\title{
The Impact of the Strategic Advertising on Luxury Fashion Brands with Social Influences
}

\author{
Jin-Hui Zheng, ${ }^{1}$ Bin Shen, ${ }^{1}$ Pui-Sze Chow, ${ }^{1}$ and Chun-Hung Chiu ${ }^{2}$ \\ ${ }^{1}$ Business Division, Institute of Textiles and Clothing, The Hong Kong Polytechnic University, Hung Hom, Kowloon, Hong Kong \\ ${ }^{2}$ Sun Yat-sen Business School, Sun Yat-sen University, Guangzhou 510275, China
}

Correspondence should be addressed to Bin Shen; binshenjerry@gmail.com

Received 6 December 2012; Accepted 23 January 2013

Academic Editor: Tsan-Ming Choi

Copyright (c) 2013 Jin-Hui Zheng et al. This is an open access article distributed under the Creative Commons Attribution License, which permits unrestricted use, distribution, and reproduction in any medium, provided the original work is properly cited.

It is well known that purchase of luxury fashion brands is strongly influenced by social needs such as the need for uniqueness and the need of conformity. The existence of these two competing social needs separates customers into two groups who exhibit different buying behaviors. This paper concerns the impacts of such social influences between different consumer groups on pricing and advertising strategies of luxury fashion brands with penalty of insufficient advertising. We start by considering different advertising allocation strategies and derive the corresponding local optimal pricing and advertising allocation policies, through which the global optimal policy that maximizes the company's profit can be obtained. Important insights on strategic advertising for luxury fashion brands are discussed.

\section{Introduction}

With the spectacular growth of consumption in its market, luxury fashion has become an area of growing interest to practitioners as well as academicians. Due to its high-price positioning, luxury fashion was used to be out of reach of mass consumption; however, luxury fashion brands are now developing strategies to manage broader diversity of consumer preferences for increasing profits. Luxury fashion brands such as Chanel, Christian Dior, Gucci, and Louis Vuitton have been employing a variety of branding strategies to tackle the problem of being out of reach of mass consumption, particularly in Asian countries such as China, Japan and Korea (notice that for many luxury fashion brands, consumers from China are critical $[1,2]$, and their conspicuous behaviors are very influential for luxury fashion brands' operations).

In addition to various branding strategies such as brand extensions [3] and strategic pricing [4], advertising is deemed an effective strategy for luxury fashion brands to develop and maintain huge demand and consumption. Apart from the physical value (quality) their products provide, luxury fashion brands highly emphasize the symbolic value (prestige) their image project. As such, advertising is a common tool that luxury brands rely on in building brand salience.
Advertising influences not only the "immediate" purchase but also the long-term brand equity of the luxury brand. Advertising, along with personal experience, is an undeniable force in creating brand equity [5]. One mechanism of creating brand equity via advertising is by creating and enhancing brand image.

Meanwhile, it is well known that consumption is strongly influenced by social needs such as prestige and self-image $[6,7]$. For luxury fashion brands, consumers are influenced by two competing social needs: the need for uniqueness and the countervailing need for conformity in purchase $[8,9]$. Accordingly, a luxury fashion brand needs to reconcile the potential tradeoffs between exclusivity and accessibility on target advertising groups. On the one hand, exclusivity makes a brand out of ordinary as well as projects a positive brand association of user profiles; on the other hand, accessibility provides sufficient sales and profits [10,11].

Recognition of the social need for uniqueness and that for conformity also implies the existence of two types of consumers, namely, the elites and the majority masses. The elites are usually more fashion conscious and would like to distinguish themselves from the masses in consumption, whilst the masses seek to emulate the choices of the elites (see [12-14] for the details). In this paper, following Zheng et al. [14], we refer to the elite consumers and the majority masses as the leader 
group (LG) and the follower group (FG), respectively. The former group usually plays a leadership role in the fashion trend, whereas the latter one is usually following the purchase of LG.

In light of the different buying behaviors between the two groups of consumers, luxury fashion brands must target each of them effectively and efficiently with proper allocation the limited advertising budget in order to obtain its best payoff. Take the classic high-end British fashion brand Burberry as an example. Burberry holds its own fashion shows in Milan every year to attract its elite customers. The brand also strengthens the social interaction with its mass customers; it is now the leading luxury fashion brand on Facebook with over one million fans. In short, Burberry utilizes the social influence for brand development, establishing a leading presence across social media platforms, and creating new communities of interest.

Zheng et al. [14] explore the impact of social influences on the optimal pricing and advertising allocation strategies of a luxury fashion brand in the presence of LG and FG, which have social influences on each other. In this paper, we extend Zheng et al. [14] with more realistic considerations. To be specific, we investigate how a luxury fashion brand allocates the limited advertising expense strategically to maximize its profit. Similar to Zheng et al. [14], we consider a retail brand owner that sells a luxury fashion product to the market that consists of two groups of consumers (LG and FG) who have social influence on each other. We consider the scenario that there is a certain "basic amount" of advertising effort for each market segment (FG, LG) in order to maintain the brand strength; otherwise the brand would suffer in the long run due to loss of goodwill from a group of consumers. To reflect that a brand has concerns on its budget allocation for sustaining the respective brand's role/positioning in the market with respect to both groups of consumers, we consider that there is a penalty cost in the form of a linear loss function for any advertising effort lower than the basic amount for each market segment.

\section{Literature Review}

In marketing science and operations management, there are a considerable amount of studies that examine optimization of analytical models with pricing and/or advertising decisions under different settings. To take into consideration of the effect of advertising, Krähmer [15] considers a model with advertising that informs the public of brand names and creates the possibility of conspicuous consumption by rendering brands as a signaling device. The author shows that advertising increases consumers' willingness to pay which provides a foundation, based on optimization behavior, for persuasive approaches to advertising. Grosset and Viscolani [16] propose a model of a firm that advertises a product in a homogeneous market, where a constant exogenous interference is present. They reveal that the optimal policy takes one of two forms: either a positive and constant advertising effort or a decreasing effort starting from a positive level and eventually reaching the zero value at a finite exit time. Ghosh and Stock [17] use a model of informative advertising to study the effect of penetration on competing advertisers' strategies and profits. Conditions under which an increase in penetration counter intuitively leads firms to increase advertising levels and enjoy higher profits are identified. In Zheng et al. [14], the authors consider that advertising has a direct impact on social needs but lack of considering the impact of the penalty of the insufficient advertising on the target groups. It is important to consider the loss from the insufficient advertising for the target group because in practices, low advertising effort on the market might have a negative impact on market demand.

It is well known that advertising on target groups can increase the group's consumption. In practice, most of the time, advertising is targeted [18, 19]. Anand and Shachar [18] study a model in which firms can target their advertisements to particular groups of consumers, and advertising is noisy. They consider the case that a particular product has a better fit with the tastes of some consumers than those of the others, and consumer-utility depends on the resulting "match" between product attributes and their tastes. Raghavan and Iyer [19] examine advertising strategy when competing firms can target their advertising effort to different groups of consumers within a market. With targeted advertising, they find that firms advertise more to consumers who have a strong preference for their product than to comparison shoppers who can be attracted to the competition. They argue that advertising less on comparison shoppers can be seen as a way for firms to endogenously increase differentiation in the market. Interestingly, they also find that target advertising leads to higher profits, regardless of the firms' ability to set targeted prices.

Our study is related to the work in social factors and conspicuous consumption $[15,20,21]$. Focusing on the conspicuous products, Krähmer [15] indicates that brands are consumed for image reasons and advertising creates a brand's image. He argues that advertising informs the public of brand names and creates the possibility of conspicuous consumption. In a price-competition framework, he shows that advertising increases consumers' willingness to pay and thus provides a foundation for determining the optimal advertising strategy. Moreover, he finds that an incumbent might strategically overinvest in advertising to deter entry, and competition might be socially undesirable. McClure and Kumcu [20] formalize the relationship between the optimal price/quantity combination and the thoroughness of conspicuous product promotions. They reveal that iterating towards the profit maximizing thoroughness of product promotion will lead to a backward bending price/quantity locus. In this paper, we study an optimal control problem in the fashion apparel industry. We establish the study which analytically shows how a firm allocates the target advertising strategically on the luxury fashion brands when facing the market demand is affected by consumer desire for exclusivity and conformity, respectively.

\section{The Model}

We consider the scenario that a company sells a fashion product to the market with two groups of customers, namely, 
the leader-group (LG) customers and the follower-group (FG) customers. Similar to Zheng et al. [14], the demands of these two groups are interdependent: a higher demand of LG induces a higher demand of FG, but a higher demand of FG implies a lower demand of LG. The unit product $\operatorname{cost}$ is $c$, and the unit retail price is $p$, where $p>c>0$. We consider the case that $c$ is exogenous, whilst $p$ is decided by the company. Both groups are price sensitive, and demands of both groups are strictly decreasing in $p$ (Chiu et al. [22]). In order to increase the sales volume of the product, the company implements advertising campaigns on LG and FG, respectively, and the total advertising resource and effort of the company spent on the advertising campaigns are denoted by $e$. Let $\lambda$ be the proportion of the effort to be spent on LG, where $\lambda \in[0,1]$. Then $(1-\lambda)$ is the proportion of the effort to be spent on FG. The advertising cost function $C(e)$ is strictly increasing in $e$, and the marginal cost of the total advertising effort is strictly increasing in $e$; that is, $d C(e) / d e>0$ and $d^{2} C(e) / d e^{2}>0$. In order to have closed-form solutions to generate more analytical insights, we consider $C(e)=h e^{2}$, where $h>0$. We denote the strategy of company with advertising and pricing by $\omega=\{e, \lambda, p\}$. We only focus on the set of finite $\omega$; that is, $\omega \in \Omega=\{0 \leq e<+\infty, 0 \leq \lambda \leq 1$, $c<p<+\infty\}$. To reflect the interdependency of the demands of different customer groups in the luxury fashion market, we adopt the following additive demand functions of LG and FG, respectively:

$$
D_{L}(\omega)=\left[V_{L}(\omega)\right]^{+}, \quad D_{F}(\omega)=\left[V_{F}(\omega)\right]^{+},
$$

where $V_{L}(\omega)=x_{L}+a \lambda e-b D_{F}(\omega)-g p, V_{F}(\omega)=x_{F}+a(1-$ $\lambda) e+\beta D_{L}(\omega)-\gamma p,[Y]^{+}=\max \{0, Y\}$, and $x_{L}, x_{F}, a, \alpha, b$, $\beta, g$, and $\gamma$ are all nonnegative.

Different from Zheng et al. [14], we further consider that certain amount of advertising is needed for each market segment in order to maintain the brand strength; otherwise the brand would suffer in the long run due to loss of goodwill from a group of consumers. We hence consider the following linear loss functions (Marinelli [23]) for insufficient advertising for LG and FG: $\Lambda_{L}(e)=m[T-\lambda e]^{+}$and $\Lambda_{F}(e)=\mu[\tau-$ $(1-\lambda) e]^{+}$, respectively, where $T \geq 0$ and $\tau \geq 0$ are the minimum advertising effort/resources for LG and FG, respectively, and $m \geq 0$ and $\mu \geq 0$ are the marginal losses due to insufficient advertising effort/resources that are assigned for LG and FG, respectively. Accordingly, the profit of the company is

$$
\begin{aligned}
\pi_{L L}(\omega)= & (p-c) D(\omega)-C(e)-\Lambda_{L}(e)-\Lambda_{F}(e), \\
= & (p-c) D(\omega)-h e^{2}-m[T-\lambda e]^{+} \\
& -\mu[\tau-(1-\lambda) e]^{+} .
\end{aligned}
$$

In this section, our objective is to derive the optimal advertising and pricing strategy for the social influence model with linear loss function for insufficient advertising. Mathematically, we consider the following optimization model:

$$
\max _{\omega \in \Omega} \pi_{L L}(\omega)=(p-c) D(\omega)-C(e)-\Lambda_{L}(e)-\Lambda_{F}(e),
$$

where $\Omega=\{e \geq 0,0 \leq \lambda \leq 1, p>c\}$. (SILL stands for Social Influence with Linear (L)oss penalty for insufficient advertising.)

Denote the optimal solution of (P-SILL) by $\omega^{*}=\left\{e^{*}\right.$, $\left.\lambda^{*}, p^{*}\right\}$. Because of the presence of the penalty functions of insufficient advertising, there exist some situations that $\pi_{L L}\left(\omega^{*}\right)<0$; that is, the company is not profitable. In this paper, we only consider the situations that the company is profitable; that is, there exist some feasible $\omega$ that $\pi_{L L}(\omega)>0$. Similar to Zheng et al. [14], we have the following assumption.

Assumption 1. $x_{L}>g c$ and $x_{F}>\gamma c$.

We employ Assumption 1 to make sure that there are positive product demands in both market segments when the product is sold at production cost, and the social influences are not considered.

Notice that since $\Lambda_{L}(e)$ and $\Lambda_{F}(e)$ are nondifferentiable at $\lambda e=T$ and $(1-\lambda) e=\tau$, respectively, $\pi_{L L}(\omega)$ is non-differentiable at $e=T / \lambda$ and $e=\tau /(1-\lambda)$.

To deal with the fact that $\pi_{L L}(\omega)$ is non-differentiable at some points, we consider the following four exclusive strategies in determining the optimal advertising and pricing policy for the company.

Strategy 1. Advertising effort assigned to both market segments are sufficient; that is, $\lambda e \geq T$ and $(1-\lambda) e \geq \tau$. In this case, we have $e \geq T+\tau$.

Strategy 2. Advertising effort assigned to LG is sufficient but that to FG is insufficient; that is, $\lambda e \geq T$ and $(1-\lambda) e<\tau$. In this case, we have $e \geq T$.

Strategy 3. Advertising effort assigned to LG is insufficient but that to FG is sufficient; that is, $\lambda e<T$ and $(1-\lambda) e \geq \tau$. In this case, we have $e \geq \tau$.

Strategy 4. Advertising effort assigned to both market segments are insufficient, that is, $\lambda e<T$ and $(1-\lambda) e<\tau$. In this case, we have $e<T+\tau$.

Here, we consider the marketing strategy that the company sells the product to both FG and LG; in other words, we assume $D_{L}(\omega)>0$ and $D_{F}(\omega)>0$. To facilitate presentation, we use $j=1,2,3,4$ to represent strategy $j$. All proofs of propositions are relegated to the Appendix.

In this paper, we focus on deriving the local optimal advertising and pricing policies, as well as the respective associated necessary conditions (and sufficient conditions as well if it can be solved out analytically) for local optimality for individual strategies. (Due to the complexity of the problem, it is very difficult to obtain the necessary and sufficient conditions for optimality for every strategy. Therefore, we mainly focus on exploring the necessary conditions for optimality.) For any given market parameters, the company can check the necessary conditions for each strategy. If any one of the necessary conditions of a particular strategy does not hold, then the local optimal of that particular strategy does not exist. In other words, the necessary conditions can be used to screen out the strategies that never provide $\omega^{*}$ (the global 
optimal solution). Afterwards, the company can calculate the corresponding company's profits amongst the strategies with local optimal policies satisfying the necessary conditions to identify the global optimal strategy, which is the one that gives the maximum profit to the company.

The total demand of the product is

$$
D(\omega)=\frac{\left(B-G(p-c)+\alpha(1-b) e+\lambda N_{I} e\right)}{(1+b \beta)}
$$

and the associated company's profit is

$$
\begin{aligned}
\pi_{L L}(\omega)= & \frac{\left\{-G(p-c)^{2}+(p-c)\left[B+\alpha(1-b) e+\lambda N_{I} e\right]\right\}}{(1+b \beta)} \\
& -h e^{2}-m[T-\lambda e]^{+}-\mu[\tau-(1-\lambda) e]^{+}
\end{aligned}
$$

where $G=(1-b) \gamma+(1+\beta) g, B=X-G c$, and $N_{I}=a(1+$ $\beta)-\alpha(1-b)$.

3.1. Strategy 1. According to the basic conditions of Strategy 1 , the company's profit is

$$
\begin{aligned}
\pi_{L L}(\omega)= & \frac{-G(p-c)^{2}+(p-c)\left[B+\alpha(1-b) e+\lambda N_{I} e\right]}{1+b \beta} \\
& -h e^{2} .
\end{aligned}
$$

We first study the optimal $\lambda$ for Strategy 1.

Proposition 2. For Strategy 1, (a) $\lambda^{*}=T /(T+\tau)$ ife $=T+\tau$; (b) $\lambda^{*}=1-\tau /$ e if $N_{I}>0$ and $e>T+\tau$; (c) $\lambda^{*}=T / e$ if $N_{I}<0$ and $e>T+\tau$; (d) $\lambda^{*}$ can take any value which satisfies $\left(1-\lambda^{*}\right) e \geq \tau$ and $\lambda^{*} e \geq T$ if $N_{I}=0$.

Proposition 2 shows that the value of $\lambda^{*}$ takes different forms under various situations. Specifically, if $e=T+\tau$, then $\lambda^{*}=T /(T+\tau)$, and hence $\lambda^{*} e=T$ and $\left(1-\lambda^{*}\right) e=\tau$. On the one hand, if the company wants to assign the minimum total advertising effort, the optimal advertising effort assigned to individual market segments is just the minimum effort in individual market segments. On the other hand, if the company wants to assign a greater advertising effort (i.e., $e>$ $T+\tau$ ), then the company should check the value of $N_{I}$ first. If $N_{I} \geq 0$, then $\lambda^{*}=1-\tau / e$, in turn $\lambda^{*} e>T$ and $\left(1-\lambda^{*}\right) e=\tau$. Equivalently, the advertising effort assigned to LG is higher than the minimum requirement, but the advertising effort assigned to FG is just sufficient for $e>T+\tau$ and $N_{I} \geq 0$. If $N_{I} \leq 0$, then $\lambda^{*} e=T$ and $\left(1-\lambda^{*}\right) e>\tau$. (Noting that $\lambda^{*}$ can take any value which satisfies $\left(1-\lambda^{*}\right) e \geq \tau$ and $\lambda^{*} e \geq T$ if $N_{I}=0$. However, for simplicity, we only demonstrate here the two special cases: $\lambda^{*}$ satisfies $\left(1-\lambda^{*}\right) e=$ $\tau$, and $\lambda^{*}$ satisfies $\lambda^{*} e=T$, for $N_{I}=0$.) Therefore, the advertising effort assigned to $F G$ is higher than the minimum requirement, but the advertising effort assigned to LG is just sufficient. As a remark, $N_{I}$ represents the sensitivity of $\lambda$ to product demand. Therefore, a bigger $\lambda$ advances product demand as well as company's profit if $N_{I}$ is positive, a smaller $\lambda$ advances product demand as well as company's profit if $N_{I}$ is negative, and the value of $\lambda$ does not affect product demand and company's profit if $N_{I}=0$.

Following Proposition 2, we further consider three substrategies of Strategy 1:

$$
\begin{aligned}
& \text { Strategy } 1(\mathrm{a}): \lambda^{*}=T /(T+\tau) \text { and } e^{*}=T+\tau ; \\
& \text { Strategy } 1(\mathrm{~b}): \lambda^{*}=1-\tau / e^{*}, N_{I} \geq 0 \text { and } e^{*}>T+\tau \text {; } \\
& \text { and } \\
& \text { Strategy } 1(\mathrm{c}): \lambda^{*}=T / e^{*}, N_{I} \leq 0 \text { and } e^{*}>T+\tau \text {. }
\end{aligned}
$$

Notice that the above-mentioned conditions are specific for the associated substrategies. In particular, they satisfy the following three basic conditions of Strategy 1 : $\lambda e \geq T$, (1$\lambda) e \geq \tau$, and $e \geq T+\tau$. We now need to check whether they fulfill the remaining basic conditions of Strategy 1, namely: $D_{L}(\omega)>0$ and $D_{F}(\omega)>0$.

Denoted by $\omega_{I .1(i)}^{*}$ the local optimal advertising and pricing policy for Strategy $1(i)$, where $i=a, b, c$. Next, we explore the local optimal advertising and pricing policies for each substrategy of Strategy 1.

Strategy 1(a). Specific conditions: $D_{L}(\omega)>0, D_{F}(\omega)>0$, $\lambda^{*}=T /(T+\tau)$, and $e^{*}=T+\tau$.

By putting $e^{*}=T+\tau$ and $\lambda^{*}=T /(T+\tau)$ into (5), the company's profit for Strategy 1(a) becomes

$$
\begin{aligned}
\pi_{L L}(\omega)= & \frac{-G(p-c)^{2}}{1+b \beta} \\
& +\frac{(p-c)[B+a(1+\beta) T+\alpha(1-b) \tau]}{1+b \beta} \\
& -h(T+\tau)^{2} .
\end{aligned}
$$

Notice that right hand side of (5) depends on $p$ only.

Proposition 3. For Strategy 1(a), the local optimal advertising and pricing policy exists only if (i) $D_{L}\left(\omega_{I .1(a)}^{*}\right)>0$ and $D_{F}\left(\omega_{I .1(a)}^{*}\right)>0$; (ii) $G>0$; and (iii) $B+a(1+\beta) T+\alpha(1-b) \tau>0$. Moreover, if the local optimal advertising and pricing policy for Strategy 1(a) exists, then it is unique and is given by

$$
\begin{aligned}
\omega_{I .1(a)}^{*}= & \left\{e_{I .1(a)}^{*}=T+\tau, \lambda_{I .1(a)}^{*}=\frac{T}{(T+\tau)},\right. \\
p_{I .1(a)}^{*} & \left.=\frac{B+a(1+\beta) T+\alpha(1-b) \tau}{2 G}+c\right\},
\end{aligned}
$$

$\pi_{L L}\left(\omega_{I .1(a)}^{*}\right)=\frac{[B+a(1+\beta) T+\alpha(1-b) \tau]^{2}}{4 G(1+b \beta)}-h(T+\tau)^{2}$.

The necessary conditions for the existence of $\omega_{I .1(a)}^{*}$ are shown in Proposition 3. Specifically, conditions in Proposition 3(i) are the basic conditions for any substrategy of 
Strategy 1. The condition in Proposition 3(ii) ensures that the company's profit function is concave in $p$, whereas the one in Proposition 3(iii) ensures that the constraint $p>c$ is satisfied. Moreover, Proposition 3 shows the explicit formulas of the local optimal advertising and pricing policy (if it exists) and the associated company's profit for Strategy 1(a).

By considering the sensitivity of $T$ to $e_{I .1(a)}^{*}, \lambda_{I .1(a)}^{*}$, and $p_{I .1(a)}^{*}$, we find that $e_{I .1(a)}^{*}, \lambda_{I .1(a)}^{*}$ and $p_{I .1(a)}^{*}$ are all strictly increasing in $T$ (because $d e_{I .1(a)}^{*} / d T=1>0, d \lambda_{I .1(a)}^{*} / d T=$ $\tau /(T+\tau)^{2}>0$, and $\left.d p_{I .1(a)}^{*} / d T=a(1+\beta) /(2 G)>0\right)$. In other words, a bigger $T$ induces a higher optimal total advertising effort, a bigger optimal proportion of advertising effort allocated to LG, and a higher local optimal retail price of the product. On the other hand, $e_{I .1(a)}^{*}$ is strictly increasing in $\tau$, and $\lambda_{I .1(a)}^{*}$ is strictly decreasing in $\tau$. Besides, $p_{I .1(a)}^{*}$ is strictly increasing in $\tau$ for $b<1$, and $p_{I .1(a)}^{*}$ is strictly decreasing in $\tau$ for $b>1$. This shows that $T$ and $\tau$ affect $\omega_{I .1(a)}^{*}$ differently. Interestingly, the values of $m$ and $\mu$ do not affect $\omega_{I .1(a)}^{*}$ and $\pi_{L L}\left(\omega_{I .1(a)}^{*}\right)$. An intuitive reason for this is that the advertising effort assigned to both market segments is sufficient, so the penalties ( $m$ and $\mu$ ) for insufficient advertisings can be ignored.

Strategy 1(b). Specific conditions: $D_{L}(\omega)>0, D_{F}(\omega)>0$, $\lambda^{*}=1-\tau / e^{*}, N_{I} \geq 0$, and $e^{*}>T+\tau$.

By putting $\lambda^{*}=1-\tau / e^{*}$ into (5), the profit of the company for Strategy $1(\mathrm{~b})$ becomes

$$
\begin{aligned}
\pi_{L L}(\omega)= & \frac{-G(p-c)^{2}+(p-c)\left[B+a(1+\beta) e-\tau N_{I}\right]}{1+b \beta} \\
& -h e^{2} .
\end{aligned}
$$

Proposition 4. For Strategy 1(b), the local optimal advertising effort (as a function of $p$ ) is given by

$$
e_{I .1(b)}^{*}(p)=\frac{a(1+\beta)}{2 h(1+b \beta)}(p-c),
$$

and $e_{I .1(b)}^{*}(p)$ is strictly increasing in $p$.

Proposition 4 asserts that, for Strategy 1(b), a higher retail price induces a high optimal advertising effort. Results of Proposition 4 are not surprising especially for luxury products. Advertising usually provides surpluses to luxury products, which are reflected by a higher retail price of the luxury product.

Proposition 5. Let $Y=4 h G(1+b \beta)-a^{2}(1+\beta)^{2}$. Then, for Strategy 1(b), the local optimal advertising and pricing policy exists only if (i) $N_{I} \geq 0, D_{L}\left(\omega_{I .1(b)}^{*}\right)>0$ and $D_{F}\left(\omega_{I .1(b)}^{*}\right)>0$; (ii) $Y>0$; (iii) $B>Y(T+\tau) /[a(1+\beta)]+N_{I} \tau$. Moreover, if the local optimal advertising and pricing policy for Strategy 1(b) exists, then it is unique and is given by

$$
\begin{gathered}
\omega_{I .1(b)}^{*}=\left\{e_{I .1(b)}^{*}, \lambda_{I .1(b)}^{*}, p_{I .1(b)}^{*}\right\}, \\
\pi_{L L}\left(\omega_{I .1(b)}^{*}\right)=\frac{h\left(B-N_{I} \tau\right)^{2}}{Y},
\end{gathered}
$$

where $e_{I .1(b)}^{*}=a(1+\beta)\left(B-N_{I} \tau\right) / Y, \lambda_{I .1(b)}^{*}=1-\tau / e_{I .1(b)}^{*}$, and $p_{I . i(b)}^{*}=c+2 h\left(B-N_{I} \tau\right)(1+b \beta) / Y$.

The necessary conditions for $\omega_{I .1(b)}^{*}$ being finite are shown in Proposition 5. Specifically, conditions in Proposition 5(i) are the specific conditions of Strategy 1(b). The condition in Proposition 5(ii) ensures that the profit function of the company for Strategy (b) is concave in $p$. The condition in Proposition 5(iii) ensures $e>T+\tau$ which is another basic condition for Strategy 1(b). Notice that $p_{I .1(b)}^{*}>c$ if and only if $B>N_{I} \tau$. Because $B>Y(T+\tau) /[a(1+\beta)]+N_{I} \tau$ implies that $B>N_{I} \tau$, the condition in Proposition 5(iii) does cover the constraint $p>c$. Moreover, Proposition 5 provides the explicit formulas of the local optimal advertising and pricing policy (if it exists) and the associated company's profit for Strategy 1(b).

According to Proposition 5, $e_{I .1(a)}^{*}, \lambda_{I .1(a)}^{*}, p_{I .1(a)}^{*}$, and $\pi_{L L}\left(\omega_{I .1(b)}^{*}\right)$ are decreasing in $\tau\left(d \lambda_{I .1(a)}^{*} / d T=-Y B / a(1+\right.$ $\beta)\left(B-N_{I} \tau\right)^{2}<0$, and the other derivatives are trivial, but independent of $T$. In other words, for Strategy 1(b), a bigger $\tau$ induces a lower advertising effort, a smaller portion of advertising effort allocated to LG, and a lower optimal retail price of the product. However, $T$ does not affect $e_{I .1(a)}^{*}, \lambda_{I .1(a)}^{*}$, $p_{I .1(a)}^{*}$, and $\pi_{L L}\left(\omega_{I .1(b)}^{*}\right)$.

Finally, similar to Strategy $1(\mathrm{a})$, the value of $m$ and $\mu$ do not affect $\omega_{I .1(b)}^{*}$ and $\pi_{L L}\left(\omega_{I .1(b)}^{*}\right)$, as the advertising budgets assigned to both market segments are sufficient, and hence the penalties $m$ and $\mu$ for insufficient advertisings can be ignored for Strategy 1(b).

Strategy 1(c). Specific conditions: $D_{L}(\omega)>0, D_{F}(\omega)>0$, $N_{I} \leq 0, e^{*}>T+\tau$, and $\lambda^{*}=T / e^{*}$.

First of all, $N_{I} \leq 0$ is equivalent to $a(1+\beta) \leq \alpha(1-$ $b)$ which implies $b<1$ as $a(1+\beta)>0$. Then, by putting $\lambda^{*}=T / e^{*}$ into (5), the profit of the company for Strategy 1(c) becomes

$$
\begin{aligned}
\pi_{L L}(\omega)= & \frac{-G(p-c)^{2}+(p-c)\left[B+\alpha(1-b) e+T N_{I}\right]}{1+b \beta} \\
& -h e^{2} .
\end{aligned}
$$

Similar to Strategy $1(\mathrm{~b})$, we obtain the following results for Strategy 1(c). 
Proposition 6. Let $Z=4 h G(1+b \beta)-\alpha^{2}(1-b)^{2}$. Then, for Strategy 1(c), (a) the local optimal advertising effort (as a function of $p$ ) is given by

$$
e_{I .1(c)}^{*}(p)=\frac{\alpha(1-b)}{2 h(1+b \beta)}(p-c),
$$

and $e_{I .1(c)}^{*}(p)$ is strictly increasing in $p$.

(b) The local optimal advertising and pricing strategy exists only if (i) $N_{I} \leq 0, D_{L}\left(\omega_{I .1(c)}^{*}\right)>0$ and $D_{F}\left(\omega_{I .1(c)}^{*}\right)>0$; (ii) $Z>0$; (iii) $B>Z(T+\tau) /[\alpha(1-b)]-N_{I} T$. Moreover, if the local optimal advertising and pricing policy for Strategy 1(c) exists, then it is unique and is given by

$$
\begin{gathered}
\omega_{I .1(c)}^{*}=\left\{e_{I .1(c)}^{*}, \lambda_{I .1(c)}^{*}, p_{I .1(c)}^{*}\right\}, \\
\pi_{L L}\left(\omega_{I .1(c)}^{*}\right)=\frac{h\left(B+N_{I} T\right)^{2}}{Z},
\end{gathered}
$$

where $e_{I .1(c)}^{*}=\alpha(1-b)\left(B+N_{I} T\right) / Z, \lambda_{I .1(c)}^{*}=T Z / \alpha(1-b)(B+$ $\left.N_{I} T\right)$, and $p_{I .1(c)}^{*}=2 h\left(B+N_{I} T\right)(1+b \beta) / Z+c$.

Similar to Propositions 4 and 5 for Strategy 1(b), Proposition 6(a) shows that the optimal advertising effort is increasing in the optimal retail price for Strategy 1(c), and the necessary conditions for $\omega_{I .1(c)}^{*}$ being finite are shown in Proposition 6(b). Specifically, conditions in Proposition 6(b)(i) are the specific conditions of Strategy 1(c). The condition in Proposition 6(b)(ii) ensures that the profit function of the company for Strategy $1(\mathrm{c})$ is concave in $p$, whereas the one in Proposition 6(b)(iii) ensures $e>T+\tau$, which is another basic condition for Strategy 1(c). Notice that $p_{I . i(c)}^{*}>c$ if $B>Z(T+\tau) /[\alpha(1-b)]-N_{I} T$. Moreover, Proposition 6(b) provides the explicit formulas of the local optimal advertising and pricing policy (if it exists) and the associated company's profit for Strategy 1(c).

According to Proposition 6, $\pi_{L L}\left(\omega_{I .1(c)}^{*}\right), e_{I .1(c)}^{*}$, and $p_{I .1(c)}^{*}$ are decreasing in $T$, while $\lambda_{I .1(c)}^{*}$ is increasing in $T$. This shows that the company will spend less on advertising, will be more focused on LG, and will set a lower retail price of the product when $T$ increases. Although there is less expenditure spent on advertising, the company still loses profit due to a lower retail price of the product offered to the customers. On the other hand, $e_{I .1(c)}^{*}, \lambda_{I .1(c)}^{*}$, and $p_{I .1(c)}^{*}$ are independent of $\tau$. Similarly, these findings are caused by the technical issue, as $T$ does not include in any specific condition of Strategy 1(c).

Finally, similar to Strategies 1(a) and 1(b), the values of $m$ and $\mu$ do not affect $\omega_{I .1(c)}^{*}$ and $\pi_{L L}\left(\omega_{I .1(c)}^{*}\right)$, as the advertising effort assigned to both market segments are sufficient, and hence the penalties $m$ and $\mu$ for insufficient advertising can be ignored for Strategy 1(c).

3.2. Strategy 2. According to the basic conditions of Strategy 2 , the company's profit is

$$
\begin{aligned}
\pi_{L L}(\omega)= & \frac{-G(p-c)^{2}+(p-c)\left[B+\alpha(1-b) e+\lambda N_{I} e\right]}{1+b \beta} \\
& -h e^{2}-\mu[\tau-(1-\lambda) e] .
\end{aligned}
$$

Proposition 7. For Strategy 2, (a) the local optimal advertising and pricing policy does not satisfy $N_{I}(p-c)<\mu(1+b \beta)$ and $e \geq T+\tau$ simultaneously; (b) $\lambda^{*}=1$ if $e=T$; (c) $\lambda^{*}=1$ if $N_{I}(p-c)>\mu(1+b \beta)$ and $e>T ;(d) \lambda^{*}=T / e$ if $N_{I}(p-c)<$ $\mu(1+b \beta)$ and $T<e<T+\tau$; and (e) multiple $\lambda^{*}$ exist if $N_{I}(p-c)=\mu(1+b \beta)$ and $e>T$.

Proposition 7 asserts that we can ignore all the cases for which $N_{I}(p-c)<\mu(1+b \beta)$ and $e \geq T+\tau$ under Strategy 2. It also shows that the value of $\lambda^{*}$ varies under different situations. Specifically, if the company wants to assign the advertising effort such that $e=T$, we have $\lambda^{*}=1$. However, if the company wants to assign the advertising effort such that $e>T$, then the company should take into account the values of $N_{I}(p-c)$ and $\mu(1+b \beta)$. In particular, if $N_{I}(p-c)>\mu(1+b \beta)$, then $\lambda^{*}=1$; if $N_{I}(p-c)<\mu(1+b \beta)$, then $\lambda^{*}=T / e$; if $N_{I}(p-c)=\mu(1+b \beta)$, then for any given $e>T$, any $\lambda$ that satisfies $(1-\lambda) e<\tau$ and $\lambda e \geq T$ is optimal for Strategy 2 . In other words, if $N_{I}(p-c)>\mu(1+b \beta)$, then the company should assign its advertising effort to LG only. If $N_{I}(p-c)<$ $\mu(1+b \beta)$, then the company should assign to LG its minimum required advertising effort $T$, whereas to FG it should assign the advertising effort less than $\tau$, the minimum required effort for the group. Lastly, if $N_{I}(p-c)=\mu(1+b \beta)$, then the company can select any $\lambda$ that satisfies $(1-\lambda) e<\tau$ and $\lambda e \geq T$. As $\lambda=1$ satisfies $(1-\lambda) e<\tau$ and $\lambda e \geq T$ for any given $>T$, we take $\lambda^{*}=1$ for $N_{I}(p-c)=\mu(1+b \beta)$. According to the previous discussions, we further consider four substrategies of Strategy 2:

$$
\begin{aligned}
& \text { Strategy 2(a):e=T, } \lambda^{*}=1 ; \\
& \text { Strategy 2(b): } N_{I}(p-c)>\mu(1+b \beta), e>T \text {, and } \lambda^{*}=1 ; \\
& \text { Strategy 2(c): } N_{I}(p-c)=\mu(1+b \beta), e>T \text {, and } \lambda^{*}=1 \text {; } \\
& \text { and } \\
& \text { Strategy } 2(\mathrm{~d}): N_{I}(p-c)<\mu(1+b \beta), T<e<T+\tau \text {, } \\
& \text { and } \lambda^{*}=T / e \text {. }
\end{aligned}
$$

The above-mentioned conditions are specific for the associated substrategies. In particular, they satisfy the following three basic conditions of Strategy 2 : $\lambda e \geq T,(1-\lambda) e<\tau$, and $e \geq T$. Next, we need to check whether they also fulfill the remaining basic conditions of Strategy 2, namely, $D_{L}(\omega)>0$ and $D_{F}(\omega)>0$.

Denote by $\omega_{I .2(i)}^{*}$, for $i=a, b, c, d$, the local optimal advertising and price policy for Strategy $2(i)$. Next, we explore the local optimal advertising and pricing policies for individual substrategies.

Strategy 2(a). Specific conditions: $D_{L}(\omega)>0, D_{F}(\omega)>0$, $e=T$, and $\lambda^{*}=1$.

By putting $e=T$ and $\lambda^{*}=1$ into (17), the profit of the company for Strategy 2(a) becomes

$$
\begin{aligned}
\pi_{L L}(\omega)= & \frac{-G(p-c)^{2}+(p-c)[B+a(1+\beta) T]}{1+b \beta} \\
& -h T^{2}-\mu \tau .
\end{aligned}
$$


Proposition 8. For Strategy 2(a), the local optimal advertising and pricing policy exists only if (i) $D_{L}\left(\omega_{I .2(a)}^{*}\right)>0$ and $D_{F}\left(\omega_{I .2(a)}^{*}\right)>0$; (ii) $G>0$; and (iii) $B+a(1+\beta) T>0$. Moreover, if the local optimal advertising and pricing policy for Strategy 2(a) exists, then it is unique and is given by

$$
\begin{gathered}
\omega_{I .2(a)}^{*}=\left\{e_{I .2(a)}^{*}=T, \lambda_{I .2(a)}^{*}=1,\right. \\
\left.p_{I .2(a)}^{*}=c+\frac{[B+a(1+\beta) T]}{(2 G)}\right\}, \\
\pi_{L L}\left(\omega_{I .2(a)}^{*}\right)=\frac{B^{2}+2 B a(1+\beta) T-Y T^{2}}{4 G(1+b \beta)}-\mu \tau .
\end{gathered}
$$

The necessary conditions for $\omega_{I .2(a)}^{*}$ being finite are shown in Proposition 8. Specifically, conditions in Proposition 8(i) are the basic conditions of Strategy 2. The condition in Proposition 8(ii) ensures that the profit function of the company for Strategy 2(a) is concave in $p$, whereas the condition in Proposition 8(iii) ensures that $p_{I .2(a)}^{*}>c$. Moreover, Proposition 8 provides the explicit formulas of the local optimal advertising and pricing strategy and the associated company's profit for Strategy 2(a). Furthermore, $e_{I .2(a)}^{*}$ and $p_{I .2(a)}^{*}$ are strictly increasing in $T$. In other words, for Strategy 2(a), a bigger $T$ induces a higher optimal advertising effort and a higher retail price of the product. On the other hand, according to Proposition 8, we know that $e_{I .2(a)}^{*}$ and $p_{I .2(a)}^{*}$ are independent of $\tau$.

Strategy 2(b). Specific conditions: $D_{L}(\omega)>0, D_{F}(\omega)>0$, $N_{I}(p-c)>\mu(1+b \beta), e>T$, and $\lambda^{*}=1$.

By putting $\lambda^{*}=1$ into (17), the profit of the company for Strategy 2(b) becomes

$\pi_{L L}(\omega)=\frac{-G(p-c)^{2}+(p-c)[B+a(1+\beta) e]}{1+b \beta}-h e^{2}-\mu \tau$.

As $p>c$ and $\mu(1+b \beta)>0, N_{I}(p-c)>\mu(1+b \beta)$ implies $N_{I}>0$.

Proposition 9. For Strategy 2(b), (a) the local optimal advertising effort is given by

$$
e_{I .2(b)}^{*}(p)=e_{I .1(b)}^{*}(p)=\frac{a(1+\beta)(p-c)}{[2 h(1+b \beta)]}
$$

and $e_{I .2(b)}^{*}(p)$ is strictly increasing in $p$.

(b) The local optimal advertising and pricing policy exists only if (i) $N_{I}>0, D_{L}\left(\omega_{I .2(b)}^{*}\right)>0$, and $D_{F}\left(\omega_{I .2(b)}^{*}\right)>0$; (ii) $Y>0$; (iii) $B>T Y /[a(1+\beta)]$; and (iv) $B \geq \mu Y /\left(2 h N_{I}\right)$.
Moreover, if the local optimal advertising and pricing policy for Strategy 2(b) exists, then it is unique and is given by

$$
\begin{gathered}
\omega_{I .2(b)}^{*}=\left\{e_{I .2(b)}^{*}, \lambda_{I .2(b)}^{*}, p_{I .2(b)}^{*}\right\}, \\
\pi_{L L}\left(\omega_{I .2(b)}^{*}\right)=\frac{h B^{2}}{Y}-\mu \tau,
\end{gathered}
$$

where $e_{I .2(b)}^{*}=a(1+\beta) B / Y, \lambda_{I .2(b)}^{*}=1$, and $p_{I .2(b)}^{*}=2 h B(1+$ $b \beta) / Y+c$.

Proposition 9(a) shows that, for Strategy 2(b), high retail price should be supported by high advertising effort of the company.

The necessary conditions for $\omega_{I .2(b)}^{*}$ being finite are shown in Proposition 9(b). Specifically, conditions in Proposition 9(b)(i) are the specific conditions of Strategy 2(b). The condition in Proposition 9(b)(ii) ensures that the profit function of the company for Strategy 2(b) is concave in $p$, whereas that in Proposition 9(b)(iii) ensures $e>T$ which is the basic condition for Strategy 2(b). The condition in Proposition 9(b)(iv) ensures that $N_{I}(p-c)>\mu(1+b \beta)$ which is another basic condition for Strategy 2(b), noting that the constraint $p_{I .2(b)}^{*}>c$ is fulfilled under the condition in Proposition 9(b)(iii). Moreover, Proposition 9(b) provides the explicit formulas of the local optimal advertising and pricing policy and the associated company's profit for Strategy 2(b). Furthermore, $\pi_{L L}\left(\omega_{I .2(b)}^{*}\right)$ is strictly decreasing in $\tau$ and $\mu$, but is independent of $T$ and $m$.

Interestingly, as shown by Proposition 9(b), $e_{I .2(b)}^{*}, \lambda_{I .2(b)}^{*}$, and $p_{I .2(b)}^{*}$ are independent of $\tau$ and $T$. These findings are different from previous findings in which both the optimal advertising effort and the optimal retail price depend on either $\tau$ or $T$. On the other hand, $e_{I .2(b)}^{*}, \lambda_{I .2(b)}^{*}$ and $p_{I .2(b)}^{*}$ are independent of $\mu$ and $m$.

Strategy 2(c). Specific conditions: $D_{L}(\omega)>0, D_{F}(\omega)>0$, $N_{I}(p-c)=\mu(1+b \beta), e>T$, and $\lambda^{*}=1$. $N_{I}>0$.

As $p>c$ and $\mu(1+b \beta)>0, N_{I}(p-c)=\mu(1+b \beta)$ implies

Proposition 10. For Strategy 2(c), (a) the local optimal advertising effort as a function of $p$ is given by

$$
e_{I .2(c)}^{*}(p)=e_{I .2(b)}^{*}(p)=\frac{a(1+\beta)(p-c)}{[2 h(1+b \beta)]},
$$

and $e_{I .2(c)}^{*}(p)$ is strictly increasing in $p$.

(b) The local optimal advertising and pricing policy exists only if (i) $N_{I}>0, D_{L}\left(\omega_{I .2(c)}^{*}\right)>0$, and $D_{F}\left(\omega_{I .2(c)}^{*}\right)>0$; and (ii) $a \mu(1+\beta)>2 h N_{I} T$. Moreover, if the local optimal advertising and pricing policy for Strategy 2(c) exists, then it is unique and is given by

$$
\begin{gathered}
\omega_{I .2(c)}^{*}=\left\{e_{I .2(c)}^{*}, \lambda_{I .2(c)}^{*}, p_{I .2(c)}^{*}\right\}, \\
\pi_{L L}\left(\omega_{I .2(c)}^{*}\right)=\frac{B \mu}{N_{I}}-\frac{Y \mu^{2}}{4 h N_{I}^{2}}-\mu \tau,
\end{gathered}
$$

where $e_{I .2(c)}^{*}=a \mu(1+\beta) /\left(2 h N_{I}\right), \lambda_{I .2(c)}^{*}=1$, and $p_{I .2(c)}^{*}=c+$ $\mu(1+b \beta) / N_{I}$. 
Proposition 10(a) asserts that, for Strategy 2(c), a high retail price should be supported by a high advertising effort of the brand.

The necessary conditions for $\omega_{I .2(c)}^{*}$ being finite are shown in Proposition 10(b). Specifically, conditions in Proposition 10(b)(i) are the specific conditions of Strategy 2(c). Proposition 10(b) also provides the explicit formulas of the local optimal advertising and pricing policy and the associated company's profit for Strategy 2(c). Furthermore, $\pi_{L L}\left(\omega_{I .2(c)}^{*}\right)$ is strictly decreasing in $\tau$ and independent of $T$ and $m$.

As shown in Proposition 10(b), $e_{I .2(c)}^{*}, \lambda_{I .2(c)}^{*}$, and $p_{I .2(c)}^{*}$ are independent of $\tau$ and $T$. These findings are similar to those for Strategy 2(b). On the other hand $e_{I .2(c)}^{*}$ and $p_{I .2(c)}^{*}$ are increasing in $\mu$ but are independent of $m$. Moreover, $\lambda_{I .2(c)}^{*}$ is independent of $\mu$ and $m$.

Strategy 2(d). Specific conditions: $D_{L}(\omega)>0, D_{F}(\omega)>0$, $N_{I}(p-c)<\mu(1+b \beta), T<e<T+\tau$ and $\lambda^{*}=T / e$.

By putting $\lambda^{*}=T / e$ into (17), the profit of the company for Strategy 2(d) becomes

$$
\begin{aligned}
\pi_{L L}(\omega)= & \frac{-G(p-c)^{2}+(p-c)\left[B+\alpha(1-b) e+T N_{I}\right]}{1+b \beta} \\
& -h e^{2}-\mu[T+\tau-e] .
\end{aligned}
$$

Proposition 11. For Strategy 2(d), (a) the local optimal advertising effort as a function of retail price $p$ is given by

$$
e_{I .2(d)}^{*}(p)=\frac{\alpha(p-c)(1-b)}{[2 h(1+b \beta)]}+\mu .
$$

(b) The local optimal advertising and pricing policy exists only if (i) $D_{L}\left(\omega_{I .2(d)}^{*}\right)>0$ and $D_{F}\left(\omega_{I .2(d)}^{*}\right)>0$; (ii) $Z>0$; (iii) $T<e_{I .2(d)}^{*}<T+\tau$; (iv) $2 h\left(B+N_{I} T\right)+\alpha \mu(1-b)>0$; and $(v)$ $N_{I}\left[2 h\left(B+N_{I} T\right)+\alpha \mu(1-b)\right] \leq \mu Z$. Moreover, if the local optimal advertising and pricing policy for Strategy 2(d) exists, then it is unique and is given by

$$
\begin{aligned}
\omega_{I .2(d)}^{*}= & \left\{e_{I .2(d)}^{*}, \lambda_{I .2(d)}^{*}, p_{I .2(d)}^{*}\right\} \\
\pi_{L L}\left(\omega_{I .2(d)}^{*}\right)= & \frac{\left[2 h B+2 h N_{I} T+\alpha \mu(1-b)\right]^{2}+\mu^{2} Z}{4 h Z} \\
& -\mu(T+\tau)
\end{aligned}
$$

where $e_{I .2(d)}^{*}=\alpha(1-b)\left[2 h\left(B+N_{I} T\right)+\alpha \mu(1-b)\right] / 2 h Z+\mu / 2 h$, $\lambda_{I .2(d)}^{*}=T / e_{I .2(d)}^{*}$, and $p_{I .2(d)}^{*}=(1+b \beta)\left[2 h\left(B+N_{I} T\right)+\alpha \mu(1-\right.$ b) $] / Z+c, \pi_{L L}(\omega)=\left(-G(p-c)^{2}+(p-c)[B+\alpha(1-b) e+\right.$ $\left.\left.T N_{I}\right]\right) /(1+b \beta)-h \mu^{2}-\mu[T+\tau-\mu]$.

Noting that $e_{I .2(d)}^{*}(p)$ is strictly increasing in $p$ only if $b<$ 1. If $b>1$, then $e_{I .2(d)}^{*}(p)$ is strictly decreasing in $p$. Therefore, Proposition 11(a) asserts that a high advertising effort of the company does not always support a high retail price and it does happen if $b>1$ for Strategy $2(\mathrm{~d})$.
The necessary conditions for $\omega_{I .2(d)}^{*}$ being finite are shown in Proposition 11(b). Specifically, conditions in Proposition 11(b)(i) are the basic conditions of Strategy 2, whilst that in Proposition 11(b)(ii) ensures that the profit function of the company for Strategy $2(\mathrm{~d})$ is concave in $p$. The condition in Proposition 11(b)(iii) ensures $T<e<T+\tau$ which is one of the specific conditions of Strategy 2(d), whereas the one in Proposition 11(b)(iv) ensures that $p_{I .2(d)}^{*}>c$. Finally, the condition in Proposition 11(b)(v) ensures that $N_{I}(p-c)<$ $\mu(1+b \beta)$ which is another specific condition of Strategy $2(\mathrm{~d})$. Moreover, Proposition 11(b) provides the explicit formulas of the local optimal advertising and pricing policy, and the associated company's profit for Strategy 2(d).

By considering the sensitivities of $e_{I .2(d)}^{*}$ and $p_{I .2(d)}^{*}$, we observe that $e_{I .2(d)}^{*}$ and $p_{I .2(d)}^{*}$ are increasing in $T$ if $N_{I}>0$, decreasing in $T$ if $N_{I}<0$, whilst they are independent of $T$ if $N_{I}=0$. In other words, a bigger $T$ induces a bigger optimal advertising effort and a higher optimal retail price for Strategy 2(d) if $N_{I}$ is positive. By contrast, a bigger $T$ induces a smaller optimal advertising effort and a smaller optimal retail price for Strategy 2(d) if $N_{I}$ is negative. Regarding the sensitivities of $\tau$ to $e_{I .2(d)}^{*}, \lambda_{I .2(d)}^{*}$, and $p_{I .2(d)}^{*}$, from Proposition 15, it is obvious that $e_{I .2(d)}^{*}, \lambda_{I .2(d)}^{*}$, and $p_{I .2(d)}^{*}$ are independent of $\tau$.

3.3. Strategy 3. According to the basic conditions of Strategy 3 , the company's profit for Strategy 3 is

$$
\begin{aligned}
\pi_{L L}(\omega)= & \frac{-G(p-c)^{2}+(p-c)\left[B+\alpha(1-b) e+\lambda N_{I} e\right]}{1+b \beta} \\
& -h e^{2}-m(T-\lambda e) .
\end{aligned}
$$

We first study the optimal solution of $\lambda$ for Strategy 3.

Proposition 12. For Strategy 3, (a) the local optimal advertising and pricing policy does not satisfy $N_{I}(p-c)>-m(1+b \beta)$ and $e \geq T+\tau$ simultaneously; (b) if $e=\tau$, then $\lambda^{*}=0$; (c) if $N_{I}(p-c)>-m(1+b \beta)$ and $\tau<e<T+\tau$, then $\lambda^{*}=1-\tau / e$; (d) if $N_{I}(p-c)<-m(1+b \beta)$ and $e>\tau$, then $\lambda^{*}=0$; (e) multiple $\lambda^{*}$ exist if $N_{I}(p-c)=-m(1+b \beta)$ and $e>T$.

Proposition 12 asserts that we can ignore all the cases for which $N_{I}(p-c)>-m(1+b \beta)$ and $e \geq T+\tau$, under Strategy 3. Moreover, Proposition 12 shows that the value of $\lambda^{*}$ varies under different situations. Specifically, if the company wants to exert its advertising effort as $e=\tau$, then the company should assign advertising effort to FG only, that is, $\lambda^{*}=0$. However, if the company wants to exert an advertising effort greater than $\tau$ (i.e., $e>\tau$ ), then the company should check the value of $N_{I}(p-c)+m(1+b \beta)$. If $N_{I}(p-c)>-m(1+b \beta)$, then $\lambda^{*}=(1-\lambda) / e$. If $N_{I}(p-c)<-m(1+b \beta)$, then $\lambda^{*}=0$. If $N_{I}(p-c)=-m(1+b \beta)$, then there exist multiple $\lambda^{*}$. In other words, if $N_{I}(p-c)<-m(1+b \beta)$, then the company should assign the advertising effort to FG only. However, if $N_{I}(p-$ c) $>-m(1+b \beta)$, then the company should assign to FG the minimum advertising resource for the group (i.e., $\tau$ ) and to LG it should assign the advertising effort less than the minimum advertising resource for the group (i.e., $T$ ). 
As $p>c$ and $m(1+b \beta)>0, N_{I}(p-c) \leq-m(1+b \beta)$ implies that $N_{I}<0$ and hence $b<1$. Furthermore, for $N_{I}(p-c)=$ $\mu(1+b \beta)$, the company can select any $\lambda$ that satisfies $(1-$ $\lambda) e<\tau$ and $\lambda e \geq T$. As $\lambda=0$ satisfies $(1-\lambda) e \geq \tau$ and $\lambda e<T$ for any given $e>T$, we take $\lambda^{*}=0$ for $N_{I}(p-c)=$ $-m(1+b \beta)$. Further to the previous discussions, we consider four substrategies of Strategy 3:

$$
\begin{aligned}
& \text { Strategy } 3(\mathrm{a}): e=\tau \text { and } \lambda^{*}=0 \\
& \text { Strategy } 3(\mathrm{~b}): N_{I}(p-c)>-m(1+b \beta), \tau<e<T+\tau, \\
& \text { and } \lambda^{*}=1-\tau / e ; \\
& \text { Strategy } 3(\mathrm{c}): b<1, N_{I}<0, N_{I}(p-c)<-m(1+b \beta), \\
& e>\tau \text {, and } \lambda^{*}=0 \text {; } \\
& \text { Strategy } 3(\mathrm{~d}): b<1, N_{I}<0, N_{I}(p-c)=-m(1+b \beta), \\
& e>\tau \text {, and } \lambda^{*}=0
\end{aligned}
$$

The above-mentioned conditions are specific to the associated substrategies. In particular, they satisfy the three basic conditions of Strategy 3: $\lambda e<T,(1-\lambda) e \geq \tau$, and $e \geq \tau$. Next we need to consider whether these substrategies also fulfill the remaining basic conditions of Strategy 3, namely, $D_{L}(\omega)>0$ and $D_{F}(\omega)>0$.

We denote by $\omega_{I .3(i)}^{*}$, for $i=a, b, c, d$, the local optimal advertising and pricing policy for Strategy 3(i). We now proceed to explore the local optimal advertising and pricing policies for individual substrategies.

Strategy 3(a). Specific conditions: $D_{L}(\omega)>0$ and $D_{F}(\omega)>0$, $e=\tau$, and $\lambda^{*}=0$.

By putting $e=\tau$ and $\lambda^{*}=0$ into (17), the profit of the company for Strategy 3(a) becomes

$$
\begin{aligned}
\pi_{L L}(\omega)= & \frac{(p-c)[B+\alpha(1-\beta) \tau]-G(p-c)^{2}}{1+b \beta} \\
& -h T^{2}-m T .
\end{aligned}
$$

Proposition 13. For Strategy 3(a), the local optimal advertising and pricing policy exists only if (i) $D_{L}\left(\omega_{I .3(a)}^{*}\right)>0$ and $D_{F}\left(\omega_{I .3(a)}^{*}\right)>0$; (ii) $G>0$; and (iii) $B+\alpha(1-b) \tau>0$. Moreover, if the local optimal advertising and pricing policy for Strategy 3 (a) exists, then it is unique and is given by

$$
\begin{gathered}
\omega_{I .3(a)}^{*}=\left\{e_{I .3(a)}^{*}=\tau, \lambda_{I .3(a)}^{*}=0,\right. \\
\left.p_{I .3(a)}^{*}=c+\frac{[B+\alpha(1-b \beta) \tau]}{(2 G)}\right\}, \\
\pi_{L L}\left(\omega_{I .3(a)}^{*}\right)=\frac{B^{2}+2 B \alpha(1-b) \tau-Z \tau^{2}}{4 G(1+b \beta)}-m T .
\end{gathered}
$$

The necessary conditions for $\omega_{I .3(a)}^{*}$ being finite are shown in Proposition 13. Specifically, conditions in Proposition 13(i) are the basic conditions of Strategy 3, and the condition in Proposition 13(ii) ensures that the profit function of the company for Strategy 3(a) is concave in $p$, whilst that in Proposition 13(iii) ensures that $p_{I .3(a)}^{*}>c$. Moreover, Proposition 13 shows the explicit formulas of the local optimal advertising and pricing policy, as well as the associated company's profit for Strategy 3(a).

For the sensitivity of $\tau$ to $e_{I .3(a)}^{*}$ and $p_{I .3(a)}^{*}$, from Proposition 13, we find that $e_{I .3(a)}^{*}$ is always strictly increasing in $\tau$. $p_{I .3(a)}^{*}$ is strictly increasing in $\tau$ only if $b<1$; if $b>1$, then $p_{I .3(a)}^{*}$ is strictly decreasing in $\tau$. In other words, for Strategy 3 (a), a bigger $\tau$ always induces a higher optimal advertising effort. A bigger $\tau$ also induces a higher retail price of the product if $b>1$, but it induces a lower retail price of the product if $b<1$.

Strategy 3(b). Specific conditions: $D_{L}(\omega)>0, D_{F}(\omega)>0$, $N_{I}(p-c)>-m(1+b \beta), \tau<e<T+\tau$, and $\lambda^{*}=1-\tau / e$.

By putting $\lambda^{*}=1-\tau / e$ into (32), the profit of the company for Strategy 3(b) becomes

$$
\begin{aligned}
\pi_{L L}(\omega)= & \frac{-G(p-c)^{2}+(p-c)\left[B+a(1+\beta) e-\tau N_{I}\right]}{1+b \beta} \\
& -h e^{2}-m(T-e+\tau) .
\end{aligned}
$$

Proposition 14. For Strategy 3(b), (a) the local optimal advertising effort as a function of retail price $p$ is given by

$$
e_{I .3(b)}^{*}(p)=\frac{a(1+\beta)}{2 h(1+b \beta)}(p-c)+\frac{m}{2 h} .
$$

Moreover, $e_{I, 3(b)}^{*}(p)$ is strictly increasing in $p$.

(b) The local optimal advertising and pricing policy exists only if (i) $D_{L}\left(\omega_{I .3(b)}^{*}\right)>0$ and $D_{F}\left(\omega_{I .3(b)}^{*}\right)>0$; (ii) $Y>0$; (iii) $B>N_{I} \tau-a m(1+\beta) /(2 h) ;(i v)(\Theta \tau-2 m G(1+b \beta)) / a(1+\beta)<$ $B<(Y T+\Theta \tau-2 m G(1+b \beta)) / a(1+\beta) ;$ and $(v)\left[2 h\left(B-N_{I} \tau\right)+\right.$ $a m(1+\beta)] N_{I}>-m Y$, where $\Theta=4 h G(1+b \beta)-a \alpha(1+\beta)(1-b)$ and $Y=4 h G(1+b \beta)-a^{2}(1+\beta)^{2}$. Moreover, (c) if the local optimal advertising and pricing policy for Strategy 3(b) exists, then it is unique and is given by

$$
\begin{gathered}
\omega_{I .3(b)}^{*}=\left\{e_{I .3(b)}^{*}, \lambda_{I .3(b)}^{*}, p_{I .3(b)}^{*}\right\}, \\
\pi_{L L}\left(\omega_{I .3(b)}^{*}\right)=\frac{\left[2 h\left(B-N_{I} \tau\right)+a m(1+\beta)\right]^{2}}{4 h^{2} Y} \\
-m(T+\tau)+\frac{m^{2}}{4 h},
\end{gathered}
$$

where $e_{I .3(b)}^{*}=a(1+\beta)\left[2 h\left(B-N_{I} \tau\right)+a m(1+\beta)\right] / 2 h Y+m / 2 h$, $\lambda_{I .3(b)}^{*}=1-\tau / e_{I .3(b)}^{*}$, and $p_{I .3(b)}^{*}=c+(1+b \beta)\left[2 h\left(B-N_{I} \tau\right)+\right.$ $\operatorname{am}(1+\beta)] / Y$.

Proposition 14(a) implies that, for Strategy 3(b), a high optimal retail price results in a high optimal advertising effort. Proposition 14(c) shows the explicit formulas of the local optimal advertising and pricing policy and the associated company's profit for Strategy 3(b). Moreover, conditions in Proposition 14(b)(i) are the basic conditions of Strategy 3; the condition in Proposition 14(b)(ii) ensures that the profit function of the company for Strategy $3(\mathrm{~b})$ is concave in $p$ whilst that in Proposition 14(b)(iii) ensures $p>c$; the 
condition in Proposition 14(b)(iv) ensures that $\tau<e<T+\tau$ which is the specific condition of Strategy 3(b). Finally, the condition in Proposition 14(b) ensures that $N_{I}(p-c)>$ $-m(1+b \beta)$, which is also the specific condition for Strategy 3 (b).

Considering the sensitivities of $T$ with respect to $e_{I .3(b)}^{*}$, $\lambda_{I .3(b)}^{*}$, and $p_{I .3(b)}^{*}$, we observe that all $e_{I .3(b)}^{*}, \lambda_{I .3(b)}^{*}$, and $p_{I .3(b)}^{*}$ depend on $N_{I}$. A bigger $T$ induces a smaller optimal advertising effort and a lower optimal retail price for Strategy 3(b) if $N_{I}$ is positive. On the contrary, a bigger $T$ induces a bigger optimal advertising effort and a higher optimal retail price for Strategy 3(b) if $N_{I}$ is negative. Finally, a bigger $T$ induces a smaller $\lambda_{I .3(b)}^{*}$ if $N_{I}=0$.

Strategy 3(c). Specific conditions: $D_{L}(\omega)>0, D_{F}(\omega)>0$, $N_{I}<0, N_{I}(p-c)<-m(1+b \beta), e>\tau$ and $\lambda^{*}=0$.

By putting $\lambda^{*}=0$ into (32), the profit of the company for Strategy 3(c) becomes

$$
\begin{aligned}
\pi_{L L}(\omega)= & \frac{-G(p-c)^{2}+(p-c)[B+\alpha(1-b) e]}{1+b \beta} \\
& -h e^{2}-m T .
\end{aligned}
$$

Proposition 15. For Strategy 3(c), (a) the local optimal advertising effort as a function of retail price $p$ is given by

$$
e_{I .3(c)}^{*}(p)=\frac{\alpha(1-b)(p-c)}{2 h(1+b \beta)},
$$

and $e_{I .3(c)}^{*}(p)$ is strictly increasing in $p$.

(b) The local optimal advertising and pricing policy exists only if (i) $N_{I}<0, D_{L}\left(\omega_{I .3(c)}^{*}\right)>0$ and $D_{F}\left(\omega_{I .3(c)}^{*}\right)>0$; (ii) $Z>$ 0; (iii) $B>\tau Z /[\alpha(1-b)]$; and (iv) $B>-m Z /\left(2 h N_{I}\right)$, where $Z=4 h G(1+b \beta)-\alpha^{2}(1-b)^{2}$. Moreover, if the local optimal advertising and pricing policy for Strategy 3(c) exists, then it is unique and is given by

$$
\begin{aligned}
\omega_{I .3(c)}^{*}= & \left\{e_{I .3(c)}^{*}=\frac{\alpha B(1-b)}{Z}, \lambda_{I .3(c)}^{*}=0,\right. \\
& \left.p_{I .3(c)}^{*}=c+\frac{2 h B(1+b \beta)}{Z}\right\}, \\
\pi_{L L}\left(\omega_{I .3(c)}^{*}\right)= & \frac{h B^{2}}{Z}-m T .
\end{aligned}
$$

Proposition 15(a) shows that, for Strategy 3(c), a higher retail price induces a higher optimal advertising effort of the brand. Proposition 15(b) shows the explicit formulas of the local optimal advertising and pricing policy and the associated company's profit for Strategy 3(c). Moreover, it also states the necessary conditions for having a finite $\omega_{I .3(c)}^{*}$. Plus, according to Proposition $15(\mathrm{~b}) e_{I .3(c)}^{*}, \lambda_{I .3(c)}^{*}$, and $p_{I .3(c)}^{*}$ are all independent of $T, \tau, m$, and $\mu$.

Strategy $3(d)$. Specific conditions: $D_{L}(\omega)>0$ and $D_{F}(\omega)>0$, $b<1, N_{I}<0, N_{I}(p-c)=-m(1+b \beta), e>\tau$, and $\lambda^{*}=0$.
Proposition 16. For Strategy 3(d), (a) the local optimal advertising effort as a function of $p$ is given by

$$
e_{I .3(d)}^{*}(p)=e_{I .3(c)}^{*}(p)=\frac{\alpha(1-b)(p-c)}{2 h(1+b \beta)},
$$

and $e_{I .3(c)}^{*}(p)$ is strictly increasing in $p$.

(b) the local optimal advertising and pricing policy exists only if (i) $N_{I}<0, D_{L}\left(\omega_{I .3(d)}^{*}\right)>0$, and $D_{F}\left(\omega_{I .3(d)}^{*}\right)>0$; (ii) $\alpha m(b-1)<2 h N_{I} \tau$. Moreover, if the local optimal advertising and pricing policy for Strategy 3(d) exists, then it is unique and is given by

$$
\begin{aligned}
\omega_{I .3(d)}^{*}= & \left\{e_{I .3(d)}^{*}=\frac{\alpha m(b-1)}{\left(2 h N_{I}\right)}, \lambda_{I .3(d)}^{*}=0,\right. \\
& \left.p_{I .3(d)}^{*}=c-\frac{m(1+b \beta)}{N_{I}}\right\}, \\
\pi_{L L}\left(\omega_{I .3(d)}^{*}\right)= & \frac{\alpha^{2} m^{2}(1-b)^{2}-4 h m\left[G m(1+b \beta)+B N_{I}\right]}{4 h N_{I}^{2}} \\
& -m T .
\end{aligned}
$$

Proposition 16(a) asserts that, for Strategy 3(d), a high retail price induces a high optimal advertising effort of the brand. Proposition 16(b) provides the explicit formulas of the local optimal advertising and pricing policy and the associated company's profit for Strategy 3(d). Moreover, the necessary conditions for having a finite $\omega_{I .3(d)}^{*}$ are shown in Proposition 16(b). Specifically, conditions in Proposition 16(b)(i) are the specific conditions of Strategy 3(d), whereas the condition in Proposition 16(b)(ii) ensures $e>\tau$, which is also the specific condition for Strategy 3(d). As shown in Proposition $16(\mathrm{~b}), e_{I .3(d)}^{*}, \lambda_{I .3(d)}^{*}$, and $p_{I .3(d)}^{*}$ are independent of $\tau, T$, and $\mu$. On the other hand, $e_{I .3(d)}^{*}$ and $p_{I .3(d)}^{*}$ are increasing in $m$.

3.4. Strategy 4. Basic conditions: $D_{L}(\omega)>0, D_{F}(\omega)>0$, $\lambda e<$ $T,(1-\lambda) e<\tau$ and $e<T+\tau$.

The company's profit for Strategy 4 is

$$
\begin{aligned}
\pi_{L L}(\omega)= & \frac{(p-c)\left[B+\alpha(1-b) e+\lambda N_{I} e\right]-G(p-c)^{2}}{1+b \beta} \\
& -h e^{2}-m T-\mu \tau+(m \lambda+\mu-\mu \lambda) e .
\end{aligned}
$$

Proposition 17. (a) When $N_{I}(p-c)>(\mu-m)(1+b \beta)$ and $T \leq e<T+\tau$, Strategy 2 dominates Strategy 4. (b) When $N_{I}(p-c)<(\mu-m)(1+b \beta)$ and $\tau \leq e<T+\tau$, Strategy 3 dominates Strategy 4. (c) When $N_{I}(p-c)=(\mu-m)(1+b \beta)$, at least one of Strategies 2 and 3 dominates Strategy 4.

Proposition 17 states the three cases under which Strategy 4 will never be the global optimal advertising and pricing strategy. Next, we focus on the remaining cases. 
Proposition 18. For Strategy 4, (a) if $N_{I}(p-c)>(\mu-m)(1+$ $b \beta)$ and $0<e<T$, then $\lambda^{*}=1$; (b) If $N_{I}(p-c)<(\mu-m)(1+$ $b \beta)$ and $0<e<\tau$, then $\lambda^{*}=0$.

Proposition 17 shows that the value of $\lambda^{*}$ differs under different situations. Note that the value of $\lambda$ can be ignored if $e=0$. If the company wants to assign advertising effort (i.e., $e>0)$, then the company should check the value of $e$ and $N_{I}(p-c)+m(1+b \beta)$. If $N_{I}(p-c)>(\mu-m)(1+b \beta)$ and $0<e<T$, then the company should assign its advertising effort to LG only. If $N_{I}(p-c)<(\mu-m)(1+b \beta)$ and $0<e<\tau$, then the company should assign its advertising effort to FG only.

We further consider the following three substrategies of Strategy 4:

$$
\begin{aligned}
& \text { Strategy } 4(\mathrm{a}): e^{*}=0 ; \\
& \text { Strategy } 4(\mathrm{~b}): N_{I}(p-c)>(\mu-m)(1+b \beta), 0<e<T \\
& \text { and } \lambda^{*}=1 ; \\
& \text { Strategy } 4(\mathrm{c}): N_{I}(p-c)<(\mu-m)(1+b \beta), 0<e<\tau \\
& \text { and } \lambda^{*}=0 \text {. }
\end{aligned}
$$

The above-mentioned conditions are specific for the associated substrategies. In particular, they satisfy the following three basic conditions of Strategy $4, \lambda e<T,(1-\lambda) e<\tau$, and $0 \leq e<T+\tau$. We proceed to check whether they also fulfill the remaining basic conditions of Strategy 4, namely: $D_{L}(\omega)>0$ and $D_{F}(\omega)>0$.

Denote by $\omega_{I .4(i)}^{*}$, for $i=a, b, c$, the local optimal advertising and price policy for Strategy $4(i)$. In the following we explore the local optimal advertising and pricing policies for individual substrategies.

Strategy 4(a). Specific conditions: $e^{*}=0$.

By putting $e^{*}=0$ into (47), the profit of the company for Strategy 4(a) becomes

$$
\pi_{L L}(\omega)=\frac{-G(p-c)^{2}+(p-c) B}{1+b \beta}-h e^{2}-m T-\mu \tau
$$

Proposition 19. For Strategy 4(a), the local optimal advertising and pricing policy exists only if (i) $D_{L}\left(\omega_{I .4(a)}^{*}\right)>0$ and $D_{F}\left(\omega_{I .4(a)}^{*}\right)>0$; (ii) $G>0$; and (iii) $B>0$. Moreover, if the local optimal advertising and pricing policy exists, then it is unique and is given by

$$
\begin{gathered}
\omega_{I .4(a)}^{*}=\left\{e_{I .4(a)}^{*}=0, \lambda_{I .4(a)}^{*}=0, p_{I .4(a)}^{*}=\frac{B}{(2 G)}+c\right\}, \\
\pi_{L L}\left(\omega_{I .4(a)}^{*}\right)=\frac{B^{2}}{4 G(1+b \beta)}-m T-\mu \tau .
\end{gathered}
$$

Similar to the other strategies, the necessary conditions for having a finite $\omega_{I .4(a)}^{*}$ are shown in Proposition 19.

Strategy 4(b). Specific conditions: $N_{I}(p-c)>(\mu-m)(1+b \beta)$, $0<e<T$, and $\lambda^{*}=1$.
By putting $\lambda^{*}=1$ into (47), the profit of the company for Strategy 4 (b) becomes

$$
\begin{aligned}
\pi_{L L}(\omega)= & \frac{-G(p-c)^{2}+(p-c)[B+a(1+\beta) e]}{1+b \beta} \\
& -h e^{2}-m T-\mu \tau+m e .
\end{aligned}
$$

Proposition 20. For Strategy 4(b), (a) the local optimal advertising effort as a function of retail price $p$ is given by

$$
e_{I .4(b)}^{*}(p)=\frac{a(1+\beta)}{2 h(1+b \beta)}(p-c)+\frac{m}{2 h}=e_{I .3(b)}^{*}(p) .
$$

Moreover, $e_{I .4(b)}^{*}(p)$ is strictly increasing in $p$.

(b) The local optimal advertising and pricing policy exists only if (i) $D_{L}\left(\omega_{I .4(b)}^{*}\right)>0$ and $D_{F}\left(\omega_{I .4(b)}^{*}\right)>0$; (ii) $Y>0$; (iii) $B>-a m(1+\beta) /(2 h)$ (condition for $p>c)$; (iv) $-2 m G(1+b \beta) /$ $a(1+\beta)<B<(T Y-2 m G(1+b \beta)) / a(1+\beta)$; and $(v) 2 h N_{I} B>$ $\mu Y-m \Theta$. Moreover, if the local optimal advertising and pricing policy for Strategy 4(b) exists, then it is unique and is given by

$$
\begin{aligned}
\omega_{I .4(b)}^{*}= & \left\{e_{I .4(b)}^{*}, \lambda_{I .4(b)}^{*}, p_{I .4(b)}^{*}\right\}, \\
\pi_{L L}\left(\omega_{I .4(b)}^{*}\right)= & \frac{h B^{2}-B a m(1+\beta)+m^{2} G(1+b \beta)}{Y} \\
& -m T-\mu \tau,
\end{aligned}
$$

where $e_{I .4(b)}^{*}=(a(1+\beta) B+2 m G(1+b \beta)) / Y, \lambda_{I .4(b)}^{*}=1$, and $p_{I .4(b)}^{*}=[2 h B+a m(1+\beta)](1+b \beta) / Y+c$

Proposition 20(a) shows that for Strategy 4(b), a higher retail price induces a higher advertising effort from the company. The necessary conditions for having a finite $\omega_{I .4(b)}^{*}$ are shown in Proposition 20(b). In particular, conditions in Proposition 20(b)(i) are the basic conditions for Strategy 4(b); the condition in Proposition 20(b)(ii) ensures that the profit function of the company for Strategy 4(b) is concave in $p$; the one in Proposition 20(b)(iii) ensures $p>c$; conditions in Proposition 20(b)(iv) and (v) ensure that $0<e<T$ and $N_{I}(p-c)>(\mu-m)(1+b \beta)$, respectively, which are the specific conditions for Strategy 4(b). Proposition 20(b) also shows the explicit formulas of the local optimal advertising and pricing policy and the associated company's profit for Strategy 4(b). Note that $e_{I .4(b)}^{*}, \lambda_{I .4(b)}^{*}$, and $p_{I .4(b)}^{*}$ are independent of $T$ and $\tau$.

Strategy 4(c). Specific conditions: $N_{I}(p-c)<(\mu-m)(1+b \beta)$, $0<e<\tau$, and $\lambda^{*}=0$.

By putting $\lambda^{*}=0$ into (47), the profit of the company for Strategy 4 (c) becomes

$$
\begin{aligned}
\pi_{L L}(\omega)= & \frac{-G(p-c)^{2}+(p-c)[B+\alpha(1-b) e]}{1+b \beta} \\
& -h e^{2}-m T-\mu \tau+\mu e .
\end{aligned}
$$


Proposition 21. For Strategy 4(c), the local optimal advertising effort as a function of retail price $p$ is given by

$$
e_{I .4(c)}^{*}(p)=\frac{\alpha(1-b)(p-c)}{2 h(1+b \beta)}+\frac{\mu}{2 h} .
$$

Note that $e_{I .4(c)}^{*}(p)$ is strictly increasing in $p$ only if $b<1$; if $b>1$, then $e_{I .4(c)}^{*}(p)$ is strictly decreasing in $p$. Therefore, Proposition 21 asserts that a higher retail price may not induce a greater advertising effort unless when $b<1$ for Strategy 4(c).

Proposition 22. For Strategy 4(c), the local optimal advertising and pricing policy exists only if (i) $D_{L}\left(\omega_{I .4(c)}^{*}\right)>0$ and $D_{F}\left(\omega_{I .4(c)}^{*}\right)>0$; (ii) $Z>0$; (iii) $2 h B+\alpha \mu(1-b)>0$; (iv) $0<2 \mu G(1+b \beta)+\alpha(1-b) B<Z \tau$; and (v) $2 h B N_{I}<\Theta \mu-Z m$. Moreover, if the local optimal advertising and pricing policy for Strategy 4(c) exists, then it is unique and is given by

$$
\begin{gathered}
\omega_{I .4(c)}^{*}=\left\{e_{I .4(c)}^{*}, \lambda_{I .4(c)}^{*}, p_{I .4(c)}^{*}\right\}, \\
\pi_{L L}\left(\omega_{I .4(c)}^{*}\right)=\frac{h B^{2}}{Z}-m T,
\end{gathered}
$$

where $e_{I .4(c)}^{*}=[2 \mu G(1+b \beta)+\alpha(1-b) B] / Z, \lambda_{I .4(c)}^{*}=0$, and $p_{I .4(c)}^{*}=(1+b \beta)[2 h B+\alpha \mu(1-b)] / Z+c$.

The necessary conditions for having a finite $\omega_{I .4(c)}^{*}$ are shown in Proposition 22. Specifically, conditions in Proposition 22(i) are the basic conditions for Strategy 4; the one in Proposition 22(ii) ensures that the profit function of the company for Strategy 4 (c) is concave in $p$; the one in Proposition 22(iii) ensures that $p>c$; finally, the conditions in Proposition 22(iv) and (v) ensure that $0<e<\tau$ and $N_{I}(p-$ $c)<(\mu-m)(1+b \beta)$, which are the specific conditions for Strategy 4(c). Moreover, Proposition 22 shows the explicit formulas of the local optimal advertising and pricing policy and the associated company's profit for Strategy 4(c). Notice that $e_{I .4(c)}^{*}, \lambda_{I .4(c)}^{*}$, and $p_{I .4(c)}^{*}$ are all independent of $T$ and $\tau$.

\section{Conclusions and Managerial Implication}

In this study, we consider the double function of advertising on (i) buying intention enhancement and (ii) long-term brand equity building. We develop the model under which a luxury fashion brand will suffer a loss when the advertising effort to different customer groups is not up to a certain level. We then derive the mechanism for the company to identify the optimal strategies and decisions in pricing and advertising budget allocation.

We summarize the major findings related to the optimal advertising strategies in the following. First, we find that when there is no penalty for insufficient advertising, it is optimal to allocate the advertising effort to a single customer group only (i.e., either LG or FG). When there is a penalty for insufficient advertising, it is more likely for the optimal policy to have the advertising effort allocated to both LG and FG. This implies that in light of the penalty for insufficient advertising, the company should take a balance when allocating the advertising effort between the two groups and should avoid being "polarized." Second, we observe that the optimal advertising effort is never decreasing in the optimal retail price when there is no penalty for insufficient advertising. However, this may not hold when there is penalty for insufficient advertising (for Strategy 2(d) with $b>1$ and Strategy $4(\mathrm{c})$ with $b>1$ ). Third, although there are 14 substrategies in total, we observe that some of them can be screened out by checking carefully their respective necessary conditions. For example, substrategies 2(b), 2(c), 3(c), and 3(d) can be screened out when $N_{I} \geq 0$, whilst substrategies 1(b), 2(b), and 2(c) can be screened out when $N_{I}<0$. Therefore, when determining the global optimal advertising and pricing policy, the company should check the value of $N_{I}$ first and then screen out substrategies that have no locally optimal and feasible solutions. Such screening can significantly reduce the computational effort required to solve the problem.

For future research, it will be important to expand the scope to explore in a supply chain context with issues such as supply chain coordination via incentive alignment schemes (see [24-29]). It will also be interesting to explore how computerized information systems support the development of luxury fashion brands [30-32]. Last but not least, it is promising to examine how the traditional logistical operational models, such as cross-docking [33] and inventory planning [34], may be employed to enhance the operational efficiency of luxury fashion brands.

\section{Appendix}

\section{Proofs}

Proof of Proposition 2. First of all, $e \geq T+\tau$ holds under Strategy 1 . For $e=T+\tau, \lambda=T /(T+\tau)$ is the only feasible solution for Strategy 1 . Therefore, $\lambda^{*}=T /(T+\tau)$ for $e=T+\tau$ under Strategy 1. For $e>T+\tau$, by taking the first-order partial derivative of (5) with respect to $\lambda$, we have $\partial \pi_{L L}(\omega) / \partial \lambda=$ $e N_{I}(p-c) /(1+b \beta)$. Therefore, for any given $p>c$ and $e>T+\tau$, if $N_{I} \geq 0$, then $\pi_{L L}(\omega)$ is increasing in $\lambda$. So $\lambda^{*}=\operatorname{Max}\{\lambda: \lambda e \geq T$ and $(1-\lambda) e \geq \tau\}$, or $\lambda^{*}=\operatorname{Max}\{\lambda:$ $\lambda \geq T / e$ and $\lambda \leq 1-\tau / e\}=1-\tau / e$. In other words, we have $\left(1-\lambda^{*}\right) e=\tau$ for $N_{I}>0$. On the other hand, if $N_{I} \leq 0$, then $\pi_{L L}(\omega)$ is decreasing in $\lambda$. So $\lambda^{*}=\operatorname{Min}\{\lambda: \lambda e \geq$ $T$ and $(1-\lambda) e \geq \tau\}$, or $\lambda^{*}=\operatorname{Min}\{\lambda: \lambda \geq T / e$ and $\lambda \leq$ $1-\tau / e\}=T / e$. Hence we have $\lambda^{*} e=T$ for $N_{I}<0$. For $N_{I}=0, \partial \pi_{L L}(\omega) / \partial \lambda=0$, so $\pi_{L L}(\omega)$ is constant for all $\lambda$. Thus $\lambda^{*}$ takes any value that satisfy $\left(1-\lambda^{*}\right) e \geq \tau$ and $\lambda^{*} e \leq T$.

Proof of Proposition 3. As the feasible set of $\omega$ for Strategy 1(a) is an open set, the local optimum for Strategy 1(a) is an interior point, if it exists. (i) Clearly, $D_{L}\left(\omega_{I .1(a)}^{*}\right)>0$ and $D_{F}\left(\omega_{I .1(a)}^{*}\right)>0$ are basic conditions for Strategy 1. (ii) By taking the first- and second-order partial derivatives of (6) with respect to $p$, we have $\partial \pi_{L L}(\omega) / \partial p=(-2 G(p-c)+[B+$ $a(1+\beta) T+\alpha(1-b) \tau]) /(1+b \beta)$ and $\partial^{2} \pi_{L L}(\omega) / \partial p^{2}=-2 \widetilde{G}$, where $\widetilde{G}=G /(1+b \beta)$. Therefore $\pi_{L L}(\omega)$ is strictly concave in $p$ if and only if $G>0$. From the first-order optimality condition, $\pi_{L L}(\omega)$ is uniquely maximized at $p_{I, 1(a)}^{*}=$ $\arg _{p}\left\{\partial \pi_{L L}(\omega) / \partial p=0\right\}=[B+a(1+\beta) T+\alpha(1-b) \tau] / 2 G+c$. 
Since $p_{I .1(a)}^{*}>c$, we obtain $B+a(1+\beta) T+\alpha(1-b) \tau>0$. Finally, by putting $\omega_{I .1(a)}^{*}$ into (6), we obtain (8).

Proof of Proposition 4. For Strategy 1(b), by taking the firstorder and second-order partial derivatives of (9) with respect to $e$, we obtain $\partial \pi_{L L}(\omega) / \partial e=a(p-c)(1+\beta) /(1+b \beta)-2 h e$, and $\partial^{2} \pi_{L L}(\omega) / \partial e^{2}=-2 h<0$. Therefore, $\partial \pi_{L L}(\omega)$ is a concave function of $e$. Then by considering $\partial \pi_{L L}(\omega) / \partial e=0$, we obtain (10). As $a(1+\beta) /[2 h(1+b \beta)]>0, e_{I, 1(b)}^{*}(p)$ is strictly increasing in $p$.

Proof of Proposition 5. As the feasible set of $\omega$ for Strategy $1(b)$ is an open set, the local optimum for Strategy 1(b) is an interior point solution, if it exists. $N_{I} \geq 0, D_{L}\left(\omega_{I . i(b)}^{*}\right)>$ 0 and $D_{F}\left(\omega_{I . i(b)}^{*}\right)>0$ are the basic conditions for Strategy $1(\mathrm{~b})$. By putting (10) into (9), we obtain $\pi_{L L}(\omega)=$ $-Y(p-c)^{2} / 4 h(1+b \beta)^{2}+\left(B-\tau N_{I}\right)(p-c) /(1+b \beta)$, and, for Strategy $1(\mathrm{~b})$, we have $\partial \pi_{L L}(\omega) / \partial p=-Y(p-c) / 2 h(1+b \beta)^{2}+$ $\left(B-\tau N_{I}\right) /(1+b \beta)$, and $\partial^{2} \pi_{L L}(\omega) / \partial p^{2}=-Y / 2 h(1+b \beta)^{2}$. Therefore, $\pi_{L L}(\omega)$ is strictly concave in $p$ if and only if $Y>0$. From the first-order optimality condition, $\pi_{L L}(\omega)$ is uniquely maximized at $p_{I .1(b)}^{*}$. Since $p_{I .1(b)}^{*}>c$, we obtain $B>N_{I} \tau$. Then by putting $p_{I .1(b)}^{*}$ into (10), we obtain $e_{I .1(b)}^{*}$. By considering $e_{I .1(b)}^{*}>T+\tau$ and $Y>0$, we obtain item (iii) of Proposition 5, which also covers $B>N_{I} \tau$. Lastly, by putting $\omega_{I .1(b)}^{*}$ into (9), we obtain (12).

Proof of Proposition 6. By taking the first-order and secondorder partial derivatives of (13) with respect to $e$, we obtain $\partial \pi_{L L}(\omega) / \partial e=\alpha(p-c)(1-b) /(1+b \beta)-2 h e$, and $\partial^{2} \pi_{L L}(\omega) /$ $\partial e^{2}=-2 h<0$. Therefore, for Strategy $1(\mathrm{c}), \pi_{L L}(\omega)$ is a concave function of $e$, and hence the optimal advertising efforts as a function of retail price $p$ is given by (14). As $\alpha(1-$ $b) /[2 h(1+b \beta)]>0, e_{I .1(c)}^{*}(p)$ is strictly increasing in $p$.

As the feasible set of $\omega$ for Strategy $1(c)$ is an open set, the local optimum for Strategy 1(c) is an interior point solution, if it exists. $N_{I} \leq 0, D_{L}\left(\omega_{I . i(c)}^{*}\right)>0$ and $D_{F}\left(\omega_{I . i(c)}^{*}\right)>0$ are basic conditions for Strategy 1(c). By putting (14) into (13), we obtain $\pi_{L L}(\omega)=-Z(p-c)^{2} / 4 h(1+b \beta)^{2}+\left(B+T N_{I}\right)(p-$ $c) /(1+b \beta)$, and we have $\partial \pi_{L L}(\omega) / \partial p=-Z(p-c) / 2 h(1+b \beta)^{2}+$ $\left(B+T N_{I}\right) /(1+b \beta)$, and $\partial^{2} \pi_{L L}(\omega) / \partial p^{2}=-Z / 2 h(1+b \beta)^{2}$.

Therefore $\pi_{L L}(\omega)$ is strictly concave in $p$ if and only if $Z>0$. By the first-order optimality condition, $\pi_{L L}(\omega)$ is uniquely maximized at $p_{I .1(c)}^{*}$. Since $p_{I .1(c)}^{*}>c$, as $Z>0$, we have $B>-N_{I} T$. Then by putting $p_{I .1(c)}^{*}$ into (14), we obtain $e_{I .1(c)}^{*}$. By considering $e_{I .1(c)}^{*}>T+\tau$, we obtain item (iii) of Proposition 6, which also covers $B>-N_{I} T$. Finally, by putting $\omega_{I .1(c)}^{*}$ into (13), we obtain (16).

Proof of Proposition 7. First of all, $e \geq T$ for Strategy 2. For $e=$ $T, \lambda=1$ is the only feasible solution for Strategy 2 . Therefore, the optimal value of $\lambda$ for Strategy 2 with $e=T$ is $\lambda^{*}=1$. For $e>T$, by taking the first-order partial derivative of (17) with respect to $\lambda$, we have $\partial \pi_{L L}(\omega) / \partial \lambda=N_{I} e(p-c) /(1+b \beta)-\mu e$ for Strategy 2. Therefore, for any given $p>c$ and $e>T$, if $N_{I}(p-c)>\mu(1+b \beta)$, then $\pi_{L L}(\omega)$ is increasing in $\lambda$. As $\lambda=1$ satisfies $\lambda e \geq T$ and $(1-\lambda) e<\tau, \lambda^{*}=1$ for $N_{I}(p-c)>$ $\mu(1+b \beta)$. If $N_{I}(p-c)<\mu(1+b \beta)$, then $\pi_{L L}(\omega)$ is decreasing in $\lambda$. Therefore, for $T<e<T+\tau$ and $N_{I}(p-c)<\mu(1+b \beta)$, the optimal $\lambda^{*}$ for Strategy 2 satisfies $\lambda^{*} e=T$. Next, let $\omega^{\prime}=$ $\left(e, \lambda^{\prime}, p\right)$ and $\omega^{\prime \prime}=\left(e, \lambda^{\prime \prime}, p\right)$, where $e \geq T+\tau, p>c, N_{I}(p-$ $c)<\mu(1+b \beta), 0 \leq \lambda^{\prime \prime}<\lambda^{\prime} \leq 1, D_{L}\left(\omega^{\prime}\right)>0, D_{F}\left(\omega^{\prime}\right)>0$, $D_{L}\left(\omega^{\prime \prime}\right)>0, D_{F}\left(\omega^{\prime \prime}\right)>0, \lambda^{\prime} e \geq T, \lambda^{\prime \prime} e \geq T,\left(1-\lambda^{\prime}\right) e<\tau$, and $\left(1-\lambda^{\prime \prime}\right)=\tau$. We have $\pi_{L L}\left(\omega^{\prime}\right)<\pi_{L L}\left(\omega^{\prime \prime}\right)$. Therefore, Strategy 1 dominates Strategy 2 for $e \geq T+\tau$ and $N_{I}(p-c)<\mu(1+b \beta)$. If $N_{I}(p-c)=\mu(1+b \beta)$, then $\pi_{L L}(\omega)$ remains constant for all values of $\lambda$. Therefore any $\lambda$ that satisfies $(1-\lambda) e<\tau$ and $\lambda e \geq T$ is an optimal solution. As there exist multiple $\lambda$ which satisfy $(1-\lambda) e<\tau$ and $\lambda e \geq T$, there exist multiple $\lambda^{*}$ for the case $N_{I}(p-c)=\mu(1+b \beta)$.

Proof of Proposition 8. The local optimum for Strategy 2(a) is an interior point solution if it exists. $D_{L}\left(\omega_{I .2(a)}^{*}\right)>0$ and $D_{F}\left(\omega_{I .2(a)}^{*}\right)>0$ are the basic conditions for Strategy 2 ; in turn they are necessary conditions for Strategy 2(a) too.By taking the first- and second-order partial derivatives of (18) with respect to $p$, we have $\partial \pi_{L L}(\omega) / \partial p=(-2 G(p-c)+[B+$ $a(1+\beta) T]) /(1+b \beta)$ and $\partial^{2} \pi_{L L}(\omega) / \partial p^{2}=-2 \widetilde{G}$, where $\widetilde{G}$ is defined in the proof of Proposition 3. Therefore, $\pi_{L L}(\omega)$ is strictly concave in $p$ if and only if $G>0$. By the first-order optimality condition, $\pi_{L L}(\omega)$ is uniquely maximized at $p_{I .2(a)}^{*}$. Since $p_{I .2(a)}^{*}>c$, we obtain item (iii) of Proposition 8. Lastly, by putting $\omega_{I .2(a)}^{*}$ into (18), we obtain (20).

Proof of Proposition 9. By taking the first-order and secondorder partial derivatives of (21) with respect to $e$, we obtain $\partial \pi_{L L}(\omega) / \partial e=a(p-c)(1+\beta) /(1+b \beta)-2 h e$, and $\partial^{2} \pi_{L L}(\omega) /$ $\partial e^{2}=-2 h<0$.

Therefore, $\partial \pi_{L L}(\omega)$ is a concave function of $e$ for Strategy 2(b). Hence the optimal advertising effort as a function of retail price $p$ is given by (22). Finally, by direct observation, $e_{I .2(b)}^{*}(p)$ is strictly increasing in $p$.

Similarly, the local optimum for Strategy 2(b) is an interior point, if it exists. Clearly, conditions $N_{I}>0, D_{L}\left(\omega_{I .2(b)}^{*}\right)>$ 0 , and $D_{F}\left(\omega_{I .2(b)}^{*}\right)>0$ are necessary for Strategy 2(b). By putting (22) into (21), we obtain $\pi_{L L}(\omega)=-Y(p-c)^{2} / 4 h(1+$ $b \beta)^{2}+B(p-c) /(1+b \beta)-\mu \tau$, and we have $\partial \pi_{L L}(\omega) / \partial p=$ $-Y(p-c) / 2 h(1+b \beta)^{2}+B /(1+b \beta)$, and $\partial^{2} \pi_{L L}(\omega) / \partial p^{2}=-Y /$ $2 h(1+b \beta)^{2}$.

Therefore, $\pi_{L L}(\omega)$ is strictly concave in $p$ if and only if $Y>$ 0 . By the first-order optimality condition, $\pi_{L L}(\omega)$ is uniquely maximized at $p_{I .2(b)}^{*}$. As $p>c$, we obtain $B>0$. By putting $p_{I .2(b)}^{*}$ into (22), we obtain $e_{I .2(b)}^{*}$. As $e>T$ for Strategy 2(b), we need $B>Y T /[a(1+\beta)]>0$, which covers the condition for $p>c$. By considering $N_{I}\left(p_{I .2(b)}^{*}-c\right)>\mu(1+b \beta)$, we obtain item (iv) of Proposition 9. Lastly, by putting $\omega_{I .2(b)}^{*}$ into (21), we obtain (24).

Proof of Proposition 10. Similarly, the local optimum for Strategy $1(\mathrm{c})$ is an interior point, if it exists. Conditions $N_{I}>0, D_{L}\left(\omega_{I .2(c)}^{*}\right)>0$ and $D_{F}\left(\omega_{I .2(c)}^{*}\right)>0$ are necessary for Strategy 2(c). As $N_{I}(p-c)=\mu(1+b \beta)$ for Strategy 2(c), we obtain $p_{I .2(c)}^{*}=c+\mu(1+b \beta) / N_{I}>c$. By putting $\omega_{I .2(c)}^{*}$ into $\pi_{L L}(\omega)$, we obtain (27). Finally, by considering $e_{I .2(c)}^{*}>T$, we obtain item (ii) of Proposition 10. 
Proof of Proposition 11. By taking the first-order and secondorder partial derivatives of (28) with respect to $e$, we obtain $\partial \pi_{L L}(\omega) / \partial e=\alpha(p-c)(1-b) /(1+b \beta)-2 h e+\mu$, and $\partial^{2} \pi_{L L}(\omega) / \partial e^{2}=-2 h<0$. Therefore, $\partial \pi_{L L}(\omega)$ is a concave function of $e$ for Strategy 2(d), and for any given $p>c$, the optimal advertising effort as a function of retail price $p$ is given by (25).

The local optimum for Strategy $1(\mathrm{~d})$ is an interior point, if it exists. $D_{L}\left(\omega_{I .2(d)}^{*}\right)>0$ and $D_{F}\left(\omega_{I .2(d)}^{*}\right)>0$ are the basic conditions of Strategy 2. By putting (29) into (28), we obtain $\pi_{L L}(\omega)=-Z(p-c)^{2} / 4 h(1+b \beta)^{2}+\left[2 h\left(B+T N_{I}\right)+\alpha \mu(1-\right.$ $b)](p-c) / 2 h(1+b \beta)-h \mu^{2}-\mu[T+\tau-\mu]$, and we have $\partial \pi_{L L}(\omega) /$ $\partial p=-Z(p-c) / 2 h(1+b \beta)^{2}+\left(2 h\left(B+T N_{I}\right)+\alpha \mu(1-b)\right) / 2 h(1+$ $b \beta)$ and $\partial^{2} \pi_{L L}(\omega) / \partial p^{2}=-Z / 2 h(1+b \beta)^{2}$.

Therefore, $\pi_{L L}(\omega)$ is strictly concave in $p$ if and only if $Z>0$. From the first-order optimality condition, $\pi_{L L}(\omega)$ is uniquely maximized at $p_{I .2(d)}^{*}$. By considering $T<e_{I .2(d)}^{*}<$ $T+\tau$, we obtain item (iii) of Proposition 11. By considering $p_{I .2(d)}^{*}>c$, we obtain item (iv) of Proposition 11. Then by considering $N_{I}\left(p_{I .2(d)}^{*}-c\right)<\mu(1+b \beta)$, we obtain item (v) of Proposition 11. Furthermore, by putting $p_{I .2(d)}^{*}$ into (29), we obtain $e_{I .2(d)}^{*}$. Finally, by putting $\omega_{I .2(d)}^{*}$ into (28), we obtain (31).

Proof of Proposition 12. First of all, $e \geq \tau$ for Strategy 3. For $e=\tau, \lambda=0$ is the only feasible solution. Therefore, the optimal value of $\lambda$ for Strategy 3 when $e=\tau$ is $\lambda^{*}=0$. For $e>\tau$, by taking the first-order partial derivative of (32) with respect to $\lambda$, we have $\partial \pi_{L L}(\omega) / \partial \lambda=N_{I} e(p-c) /(1+b \beta)+m e$.

Therefore, for any given $p>c$ and $e>\tau$, if $N_{I}(p-c)>$ $-m(1+b \beta)$, then $\pi_{L L}(\omega)$ is strictly increasing in $\lambda$. For $\tau<$ $e<T+\tau, \lambda=1-\tau / e$ is the biggest value that satisfies $\lambda e<T$ and $(1-\lambda) e \geq \tau$. Therefore, $\lambda^{*}=1-\tau / e$ for Strategy 3 if $N_{I}(p-c)>-m(1+b \beta)$ and $\tau<e<T+\tau$. Next, consider $\omega^{\prime}=\left(e, \lambda^{\prime}, p\right)$ and $\omega^{\prime \prime}=\left(e, \lambda^{\prime \prime}, p\right)$, where $e \geq T+\tau, p>c$, $N_{I}(p-c)>-m(1+b \beta), 0 \leq \lambda^{\prime}<\lambda^{\prime \prime} \leq 1, D_{L}\left(\omega^{\prime}\right)>0$, $D_{F}\left(\omega^{\prime}\right)>0, D_{L}\left(\omega^{\prime \prime}\right)>0, D_{F}\left(\omega^{\prime \prime}\right)>0, \lambda^{\prime} e<T, \lambda^{\prime \prime} e=T$, $\left(1-\lambda^{\prime}\right) e \geq \tau$, and $\left(1-\lambda^{\prime \prime}\right) \geq \tau$. We have $\pi_{L L}\left(\omega^{\prime}\right)<\pi_{L L}\left(\omega^{\prime \prime}\right)$. Therefore, Strategy 1 dominates Strategy 3 for $e \geq T+\tau$ and $N_{I}(p-c)>-m(1+b \beta)$. If $N_{I}(p-c)<-m(1+b \beta)$, then $\pi_{L L}(\omega)$ is strictly decreasing in $\lambda$. As $\lambda=0$ satisfies $\lambda e<T$ and $(1-\lambda) e \geq \tau, \lambda^{*}=0$ for Strategy 3 if $N_{I}(p-c)<-m(1+b \beta)$. If $N_{I}(p-c)=-m(1+b \beta)$, then $\pi_{L L}(\omega)$ is independent of $\lambda$ for Strategy 3 . As there are multiple $\lambda$ which satisfy $\left(1-\lambda^{*}\right) e<\tau$ and $\lambda^{*} e \geq T$, there exist multiple $\lambda^{*}$ for the case $N_{I}(p-c)=$ $-m(1+b \beta)$.

Proof of Proposition 13. Similarly, the local optimum for Strategy 3(a) is an interior point, if it exists. $D_{L}\left(\omega_{I .3(a)}^{*}\right)>0$ and $D_{F}\left(\omega_{I .3(a)}^{*}\right)>0$ are necessary for any substrategies of Strategy 3. By taking the first- and second-order partial derivatives of (33) with respect to $p$, we have $\partial \pi_{L L}(\omega) / \partial p=(-2 G(p-c)+[B+\alpha(1-b) \tau]) /(1+b \beta)$ and $\partial^{2} \pi_{L L}(\omega) / \partial p^{2}=-2 \widetilde{G}$, where $\widetilde{G}$ is defined in the proof of Proposition 3. Therefore, $\pi_{L L}(\omega)$ is strictly concave in $p$ if and only if $G>0$. From the first-order optimality condition, $\pi_{L L}(\omega)$ is uniquely maximized at $p_{I .3(a)}^{*}$. As $p>c$, we obtain item (iii) of Proposition 13. Finally, by putting $\omega_{I .3(a)}^{*}$ into (33), we obtain (34).

Proof of Proposition 14. By taking the first-order and secondorder partial derivatives of (36) with respect to $e$, we obtain $\partial \pi_{L L}(\omega) / \partial e=a(p-c)(1+\beta) /(1+b \beta)-2 h e+m$, and $\partial^{2} \pi_{L L}(\omega) / \partial e^{2}=-2 h<0$. Therefore, $\partial \pi_{L L}(\omega)$ is a concave function of $e$ for Strategy 3(b) and for any given $p>c$. Hence, the optimal advertising effort as a function of retail price $p$ is given by (37). Next, as $a(1+\beta) /[2 h(1+b \beta)]>0, e_{I .3(b)}^{*}(p)$ is strictly increasing in $p$.

Similarly, the local optimum for Strategy 1(a) is an interior point, if it exists. $D_{L}\left(\omega_{I .3(b)}^{*}\right)>0$ and $D_{F}\left(\omega_{I .3(b)}^{*}\right)>0$ are the basic conditions of Strategy 3. By putting (37) into (36), we obtain $\pi_{L L}(\omega)=-Y(p-c)^{2} / 4 h(1+b \beta)^{2}+\left[2 h\left(B-N_{I} \tau\right)+\right.$ $a m(1+\beta)](p-c) / 2 h(1+b \beta)-m(T+\tau)+m^{2} / 4 h$, and we have $\partial \pi_{L L}(\omega) / \partial p=-Y(p-c) / 2 h(1+b \beta)^{2}+\left[2 h\left(B-N_{I} \tau\right)+\right.$ $a m(1+\beta)] / 2 h(1+b \beta)$ and $\partial^{2} \pi_{L L}(\omega) / \partial p^{2}=-Y / 2 h(1+b \beta)^{2}$. Therefore, $\pi_{L L}(\omega)$ is strictly concave in $p$ if and only if $Y>0$. From the first-order optimality condition, $\pi_{L L}(\omega)$ is uniquely maximized at $p_{I .3(b)}^{*}$. By considering $p_{I .3(b)}^{*}>c$, we obtain item (iii) of Proposition 14. Similarly, by considering $\tau<$ $e_{I .3(b)}^{*}<T+\tau$ and $N_{I}\left(p_{I .3(b)}^{*}-c\right)>-m(1+b \beta)$, we obtain items (iv) and (v) of Proposition 14, respectively. By putting $p_{I .3(b)}^{*}$ into (37), we obtain $e_{I .3(b)}^{*}$. Finally, by putting (38) into (36). we obtain (39).

Proof of Proposition 15. By taking the first-order and secondorder partial derivatives of (40) with respect to $e$, we obtain $\partial \pi_{L L}(\omega) / \partial e=\alpha(1-b)(p-c) /(1+b \beta)-2 h e$, and $\partial^{2} \pi_{L L}(\omega) /$ $\partial e^{2}=-2 h<0$. Therefore, for any given $p>c, \pi_{L L}(\omega)$ is a concave function of $p$ under Strategy 3(c). Therefore, the optimal advertising effort as a function of retail price $p$ is given by (41). As $\alpha(1-b) /[2 h(1+b \beta)]>0, e_{I .3(c)}^{*}(p)$ is strictly increasing in $p$.

Similarly, the local optimum for Strategy 3(c) is an interior point, if it exists. $N_{I}<0, D_{L}\left(\omega_{I .3(c)}^{*}\right)>0$ and $D_{F}\left(\omega_{I .3(c)}^{*}\right)>$ 0 are the basic conditions for Strategy 3. By putting (41) into (40), we obtain $\pi_{L L}(\omega)=-Z(p-c)^{2} / 4 h(1+b \beta)^{2}+B(p-$ $c) /(1+b \beta)-m T$, and we have $\partial \pi_{L L}(\omega) / \partial p=-Z(p-$ c) $/ 2 h(1+b \beta)^{2}+B /(1+b \beta)$ and $\partial^{2} \pi_{L L}(\omega) / \partial p^{2}=-Z / 2 h(1+$ $b \beta)^{2}$. Therefore, $\pi_{L L}(\omega)$ is strictly concave in $p$ if and only if $Z>0$. From the first-order optimality condition, $\pi_{L L}(\omega)$ is uniquely maximized at $p_{I .3(c)}^{*}$. By considering $p_{I .3(c)}^{*}>c$, we obtain $B>0$. By considering $e_{I .3(c)}^{*}>\tau$, we obtain item (iii) of Proposition 15. By considering $N_{I}\left(p_{I .3(c)}^{*}-c\right)<-m(1+b \beta)$, we obtain $B \geq-m Z /\left(2 h N_{I}\right)>0$. Finally, by putting $\omega_{I .3(c)}^{*}$ into (40), we obtain (43).

Proof of Proposition 16. Similarly, the local optimum for Strategy $3(\mathrm{~d})$ is an interior point, if it exists. $N_{I}<0$, $D_{L}\left(\omega_{I .3(d)}^{*}\right)>0$ and $D_{F}\left(\omega_{I .3(d)}^{*}\right)>0$ are specific conditions of Strategy $3(\mathrm{~d})$. As $N_{I}(p-c)=-m(1+b \beta)$ for Strategy 3(d), we obtain $p_{I .3(d)}^{*}$. Notice that $p_{I .3(d)}^{*}>0$ as $N_{I}<0$ for Strategy 3(d). According to Proposition 23, we obtain $e_{I .3(d)}^{*}=e_{I .3(d)}^{*}\left(p_{I .3(d)}^{*}\right)$. By putting $\omega_{I .3(d)}^{*}$ into (17), we obtain (46). Finally, by considering $e_{I .3(d)}^{*}>\tau$, we obtain item (ii) of Proposition 16. 
Proof of Proposition 17. Consider $\omega^{\prime}=\left(e^{\prime}, \lambda^{\prime}, p^{\prime}\right)$ and $\omega^{\prime \prime}=$ $\left(e^{\prime}, \lambda^{\prime \prime}, p^{\prime}\right)$, where $0<\lambda^{\prime}<\lambda^{\prime \prime}<1, T \leq e^{\prime}<T+\tau, N_{I}\left(p^{\prime}-\right.$ c) $>(\mu-m)(1+b \beta), D_{L}\left(\omega^{\prime}\right)>0, D_{L}\left(\omega^{\prime \prime}\right)>0, D_{F}\left(\omega^{\prime}\right)>$ 0 , and $D_{F}\left(\omega^{\prime \prime}\right)>0$. By following the proof of Proposition 12, for any fixed $p>c$ and $T \leq e<T+\tau, \pi_{L L}(\omega)$ is strictly increasing in $\lambda$. Therefore, $\pi_{L L}\left(\omega^{\prime \prime}\right)>\pi_{L L}\left(\omega^{\prime}\right)$. However, $\omega^{\prime \prime}=\left(e^{\prime}, \lambda^{\prime \prime}, p^{\prime}\right)$ is a solution of Strategy 2 instead of Strategy 4 for $\lambda^{\prime \prime} \rightarrow 1$. Hence, the optimal $\lambda^{*}$ for Strategy 4 does not exist (i.e., Strategy 2 dominates Strategy 4 for any fixed $p>c$ and $T \leq e<T+\tau)$. This completes the proof of part (a). The proof of part (b) and part (c) are similar to the proof of part (a).

Proof of Proposition 18. First of all, $e<T+\tau$ for Strategy 4. If $e=0$, then $\lambda$ can be ignored. For $0<e<T+\tau$, by taking the first-order partial derivative of (47) with respect to $\lambda$, we have $\partial \pi_{L L}(\omega) / \partial \lambda=N_{I} e(p-c) /(1+b \beta)+(m-\mu) e$. Therefore, for any fixed $p>c$ and $0<e<T$, (a) if $N_{I}(p-c)>(\mu-m)(1+b \beta)$, then $\pi_{L L}(\omega)$ is increasing in $\lambda$. Hence the optimal value of $\lambda$ in this case is $\lambda^{*}=1$ (please note that $\lambda^{*}=1$ satisfies $\lambda e<T$ and $(1-\lambda) e<\tau$ for $0<e<T)$; (b) if $N_{I}(p-c)<(\mu-$ $m)(1+b \beta)$, then $\pi_{L L}(\omega)$ is decreasing in $\lambda$. Hence the optimal value of $\lambda$ in this case is $\lambda^{*}=0$ (please note that $\lambda^{*}=0$ satisfies $\lambda e<T$ and $(1-\lambda) e<\tau$ for $0<e<\tau)$; (c) if $N_{I}(p-$ $c)=(\mu-m)(1+b \beta)$, then $\pi_{L L}(\omega)$ is independent of $\lambda$. As there are multiple $\lambda$ which satisfy $\left(1-\lambda^{*}\right) e<\tau$ and $\lambda^{*} e<T$, for $0<e<T+\tau$, there exists multiple $\lambda^{*}$ for the case $N_{I}(p-c)=$ $(\mu-m)(1+b \beta)$.

Proof of Proposition 19. Similarly, the local optimum for Strategy 4(a) is an interior point, if it exists. Conditions $D_{L}\left(\omega_{I .4(a)}^{*}\right)>0$ and $D_{F}\left(\omega_{I .4(a)}^{*}\right)>0$ are necessary for any substrategies of Strategy 4 . By taking the first- and secondorder partial derivatives of (48) with respect to $p$, we have $\partial \pi_{L L}(\omega) / \partial p=(-2 G(p-c)+B) /(1+b \beta)$ and $\partial^{2} \pi_{L L}(\omega) /$ $\partial p^{2}=-2 \widetilde{G}$. Therefore, $\pi_{L L}(\omega)$ is strictly concave in $p$ if and only if $G>0$. From the first-order optimality condition, $\pi_{L L}(\omega)$ is uniquely maximized at $p_{I .4(a)}^{*}$. By considering $p_{I .4(a)}^{*}>c$, we obtain $B>0$. By putting $\omega_{I .4(a)}^{*}$ into (48), we obtain (50).

Proof of Proposition 20. By taking the first-order and secondorder partial derivatives of (51) with respect to $e$, we obtain $\partial \pi_{L L}(\omega) / \partial e=a(p-c)(1+\beta) /(1+b \beta)-2 h e+m$, and $\partial^{2} \pi_{L L}(\omega) / \partial e^{2}=-2 h<0$. Therefore, $\partial \pi_{L L}(\omega)$ is a concave function of $e$ for Strategy 4(b). Thus for any given $p>c$, the optimal advertising effort as a function of retail price $p$ is given by (52). According to Proposition 16, $e_{I .3(b)}^{*}(p)$ is strictly increasing in $p$. Therefore $e_{I .4(b)}^{*}(p)$, which is equal to $e_{I .3(b)}^{*}(p)$, is also strictly increasing in $p$.

Similarly, the local optimum for Strategy $4(\mathrm{~b})$ is an interior point, if it exists. Conditions $D_{L}\left(\omega_{I .4(b)}^{*}\right)>0$ and $D_{F}\left(\omega_{I .4(b)}^{*}\right)>0$ are necessary conditions for Strategy $4(\mathrm{~b})$. By putting (52) into (51), we obtain $\pi_{L L}(\omega)=-Y(p-$ c) $)^{2} / 4 h(1+b \beta)^{2}+[2 h B+a m(1+\beta)](p-c) / 2 h(1+b \beta)-m T-\mu \tau+$ $m^{2} / 4 h$, and we have $\partial \pi_{L L}(\omega) / \partial p=-Y(p-c) / 2 h(1+b \beta)^{2}+$ $(2 h B+a m(1+\beta)) / 2 h(1+b \beta)$, and $\partial^{2} \pi_{L L}(\omega) / \partial p^{2}=-Y /$ $2 h(1+b \beta)^{2}$.
Therefore, $\pi_{L L}(\omega)$ is strictly concave in $p$ if and only if $Y>0$. From the first-order optimality condition, $\pi_{L L}(\omega)$ is uniquely maximized at $p_{I .4(b)}^{*}$. By considering $p_{I .4(b)}^{*}>c$, we obtain $2 h B+a m(1+\beta)>0$. Then by putting $p_{I .4(b)}^{*}$ into (52), we obtain $e_{I .4(b)}^{*}$. By considering $N_{I}\left(p_{I .4(b)}^{*}-c\right)>(\mu-$ $m)(1+b \beta)$ and $0<e_{I .4(b)}^{*}<T$, we obtain item (iv) and item (v) of Proposition 20, respectively. Finally, by putting $\omega_{I .4(b)}^{*}$ into (51), we obtain (54).

Proof of Proposition 21. By taking the first-order and secondorder partial derivatives of (55) with respect to $e$, we obtain $\partial \pi_{L L}(\omega) / \partial e=\alpha(1-b)(p-c) /(1+b \beta)-2 h e+\mu$, and $\partial^{2} \pi_{L L}(\omega) / \partial e^{2}=-2 h<0$. Therefore, $\partial \pi_{L L}(\omega)$ is a concave function of $e$ for Strategy 4(c) for any given $p>c$. From the first-order optimality condition, the optimal advertising effort as a function of retail price $p$ is given by (50).

Proof of Proposition 22. Similarly, the local optimum for Strategy 1(a) is an interior point, if it exists. Conditions $D_{L}\left(\omega_{I .4(c)}^{*}\right)>0$ and $D_{F}\left(\omega_{I .4(c)}^{*}\right)>0$ are necessary for Strategy $4(\mathrm{c})$. By putting (56) into (55), we obtain $\pi_{L L}(\omega)=$ $-Z(p-c)^{2} / 4 h(1+b \beta)^{2}+[2 h B+\alpha \mu(1-b)](p-c) / 2 h(1+b \beta)-$ $m T-\mu \tau+\mu^{2} / 4 h$, and we have $\partial \pi_{L L}(\omega) / \partial p=-Z(p-c) /$ $2 h(1+b \beta)^{2}+(2 h B+\alpha \mu(1-b)) / 2 h(1+b \beta)$ and $\partial^{2} \pi_{L L}(\omega) / \partial p^{2}=$ $-Z / 2 h(1+b \beta)^{2}$.

Therefore $\pi_{L L}(\omega)$ is strictly concave in $p$ if and only if $Z>0$. From the first-order optimality condition, $\pi_{L L}(\omega)$ is uniquely maximized at $p_{I .4(c)}^{*}$. By considering $p_{I .4(c)}^{*}>c$, we obtain item (ii) of Proposition 22. By considering $0<e_{I .4(c)}^{*}<$ $\tau$ and $N_{I}\left(p_{I .4(c)}^{*}-c\right)<(\mu-m)(1+b \beta)$, we obtain item (iv) and item (v) of Proposition 22, respectively. Finally, by putting $\omega_{I .4(c)}^{*}$ into (55), we obtain (56).

\section{Acknowledgement}

This paper is supported by the "985 Project" of Sun Yat-Sen University (Account nos. 14000-3282101 and 14000-3281303). The authors sincerely thank the anonymous reviewers for their constructive comments on this paper.

\section{References}

[1] T. M. Choi, S. C. Liu, K. M. Pang, and P. S. Chow, "Shopping behaviors of individual tourists from the Chinese Mainland to Hong Kong," Tourism Management, vol. 29, no. 4, pp. 811-820, 2008.

[2] S. C. Liu, T. M. Choi, R. Au, and C. L. Hui, "A study on individual tourists from the Chinese mainland to Hong Kong: implications for tourism marketing in fashion," Tourism Economics, vol. 17, pp. 1287-1309, 2011.

[3] T. M. Choi, S. C. Liu, C. S. Tang, and Y. Yu, "A cross-cluster and cross-region analysis of fashion brand extensions," The Journal of the Textile Institute, vol. 102, no. 10, pp. 890-904, 2011.

[4] T. M. Choi, P. S. Chow, and T. Xiao, "Electronic price-testing scheme for fashion retailing with information updating," International Journal of Production Economics, vol. 140, pp. 396-406, 2012.

[5] D. A. Aaker and A. L. Biel, Brand Equity \& Advertising: Advertising's Role in Building Strong Brands, Lawrence Erlbaum, 1993. 
[6] T. L. Childers and A. R. Rao, "The influence of familial and peer-based reference groups on consumer decisions," Journal of Consumer Research, vol. 19, no. 2, pp. 198-211, 1992.

[7] M. Grinblatt, M. Keloharju, and S. Ikäheimo, "Social influence and consumption: evidence from the automobile purchases of neighbors," Review of Economics and Statistics, vol. 90, no. 4, pp. 735-753, 2008.

[8] M. B. Brewer, "The social self: on being the same and different at the same time," Personality and Social Psychology Bulletin, vol. 17, no. 5, pp. 475-482, 1991.

[9] K. T. Tian, W. O. Bearden, and G. L. Hunter, "Consumers' need for uniqueness: scale development and validation," Journal of Consumer Research, vol. 28, no. 1, pp. 50-66, 2001.

[10] A. M. Fionda and C. M. Moore, "The anatomy of the luxury fashion brand," Journal of Brand Management, vol. 16, no. 5-6, pp. 347-363, 2009.

[11] K. L. Keller, "Managing the growth tradeoff: challenges and opportunities in luxury branding," Journal of Brand Management, vol. 16, no. 5-6, pp. 290-301, 2009.

[12] P. Bourdieu and R. Nice, Distinction: A Social Critique of the Judgment of Taste, Harvard University Press, 1984.

[13] B. Bryson, “'Anything but heavy metal': symbolic exclusion and musical dislikes," American Sociological Review, vol. 61, no. 5, pp. 884-899, 1996.

[14] J. Zheng, C. H. Chiu, and T. M. Choi, "Optimal advertising and pricing strategies for luxury fashion brands with social influences," IEEE Transactions on Systems, Man, and Cybernetics, Part A, vol. 42, pp. 827-837, 2012.

[15] D. Krähmer, "Advertising and conspicuous consumption," Journal of Institutional and Theoretical Economics, vol. 162, no. 4, pp. 661-682, 2006

[16] L. Grosset and B. Viscolani, "Optimal dynamic advertising with an adverse exogenous effect on brand goodwill," Automatica, vol. 45, no. 4, pp. 863-870, 2009.

[17] B. Ghosh and A. Stock, "Advertising effectiveness, digital video recorders, and product market competition," Marketing Science, vol. 29, no. 4, pp. 639-649, 2010.

[18] B. N. Anand and R. Shachar, "Targeted advertising as a signal," Quantitative Marketing and Economics, vol. 7, no. 3, pp. 237-266, 2009.

[19] H. Raghavan and R. Iyer, Probabilistic First Pass Retrieval for Search Advertising: from Theory to Practice, ACM, 2010.

[20] J. McClure and E. Kumcu, "Promotions and product pricing: parsimony versus Veblenesque demand," Journal of Economic Behavior and Organization, vol. 65, no. 1, pp. 105-117, 2008.

[21] K. Yamada, "Macroeconomic implications of conspicuous consumption: a Sombartian dynamic model," Journal of Economic Behavior and Organization, vol. 67, no. 1, pp. 322-337, 2008.

[22] C. H. Chiu, J. Zheng, and T. M. Choi, "Optimal pricing and inventory decisions for fashion retailers under value-at-risk objective," in Fashion Supply Chain Management: Industry and Business Analysis, T. M. Choi, Ed., chapter 5, pp. 100-109, IGI Global, 2011.

[23] C. Marinelli, “The stochastic goodwill problem," European Journal of Operational Research, vol. 176, no. 1, pp. 389-404, 2007.

[24] B. Shen, T. M. Choi, Y. Wang, and C. K. Y. Lo, “The coordination of fashion supply chains with a risk averse supplier by the markdown money policy," IEEE Transactions on Systems, Man, and Cybernetics, 2013.

[25] N. Liu, T. M. Choi, M. C. W. Yuen, and F. Ng, "Optimal pricing, modularity and return policy under mass customization," IEEE
Transactions on Systems, Man, and Cybernetics, Part A, vol. 42, pp. 604-614, 2012.

[26] C. H. Chiu, T. M. Choi, and C. S. Tang, "Price, rebate, and returns supply contracts for coordinating supply chains with price-dependent demands," Production and Operations Management, vol. 20, no. 1, pp. 81-91, 2011.

[27] C. H. Chiu, T. M. Choi, H. T. Yeung, and Y. Zhao, "Sales rebate contracts in fashion supply chains," Mathematical Problems in Engineering, vol. 2012, Article ID 908408, 19 pages, 2012.

[28] H. J. Peng and M. H. Zhou, "Quantity discount supply chain models with fashion products and uncertain yield," Mathematical Problems in Engineering. In press.

[29] S. Costantini, G. De Gasperis, A. Provetti, and P. Tsintza, "Heuristic approach to proposal-based negotiation," Mathematical Problems in Engineering. In press.

[30] T. M. Choi and S. Sethi, "Innovative quick response programs: a review," International Journal of Production Economics, vol. 127, no. 1, pp. 1-12, 2010.

[31] T. M. Choi, P. S. Chow, and S. C. Liu, "Implementation of fashion ERP systems in China: case study, review and future challenges," International Journal of Production Economics, 2013.

[32] T. M. Choi, C. L. Hui, S. F. Ng, and Y. Yu, "Color trend forecasting of fashionable products with very few historical data," IEEE Transactions on Systems, Man, and Cybernetics, Part C, vol. 42, pp. 1003-1010, 2012.

[33] Z. H. Hu, Y. X. Zhao, and T. M. Choi, "Vehicle routing problem for fashion supply chains with cross-docking," Mathematical Problems in Engineering. In press.

[34] T. M. Choi and C. H. Chiu, "Mean-downside-risk and meanvariance newsvendor models: implications for sustainable fashion retailers," International Journal of Production Economics, vol. 135, pp. 552-560, 2012. 


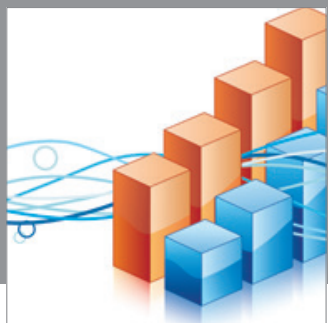

Advances in

Operations Research

mansans

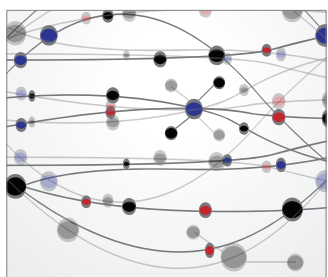

The Scientific World Journal
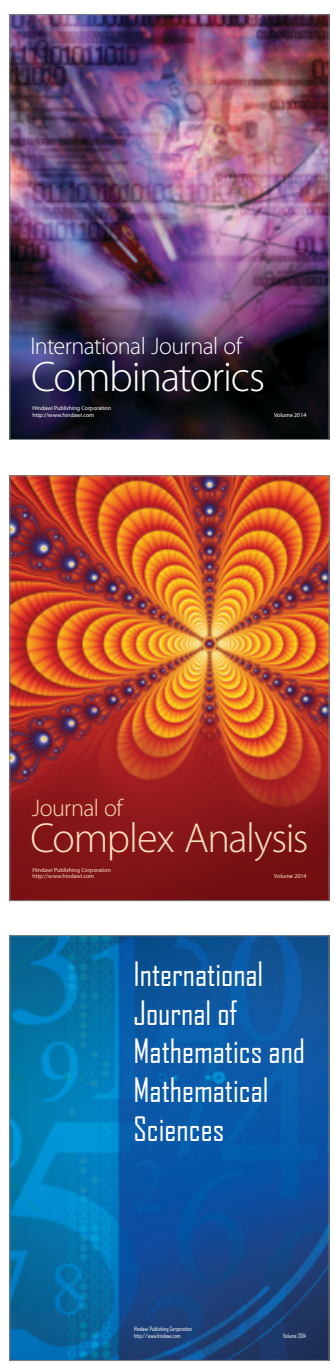
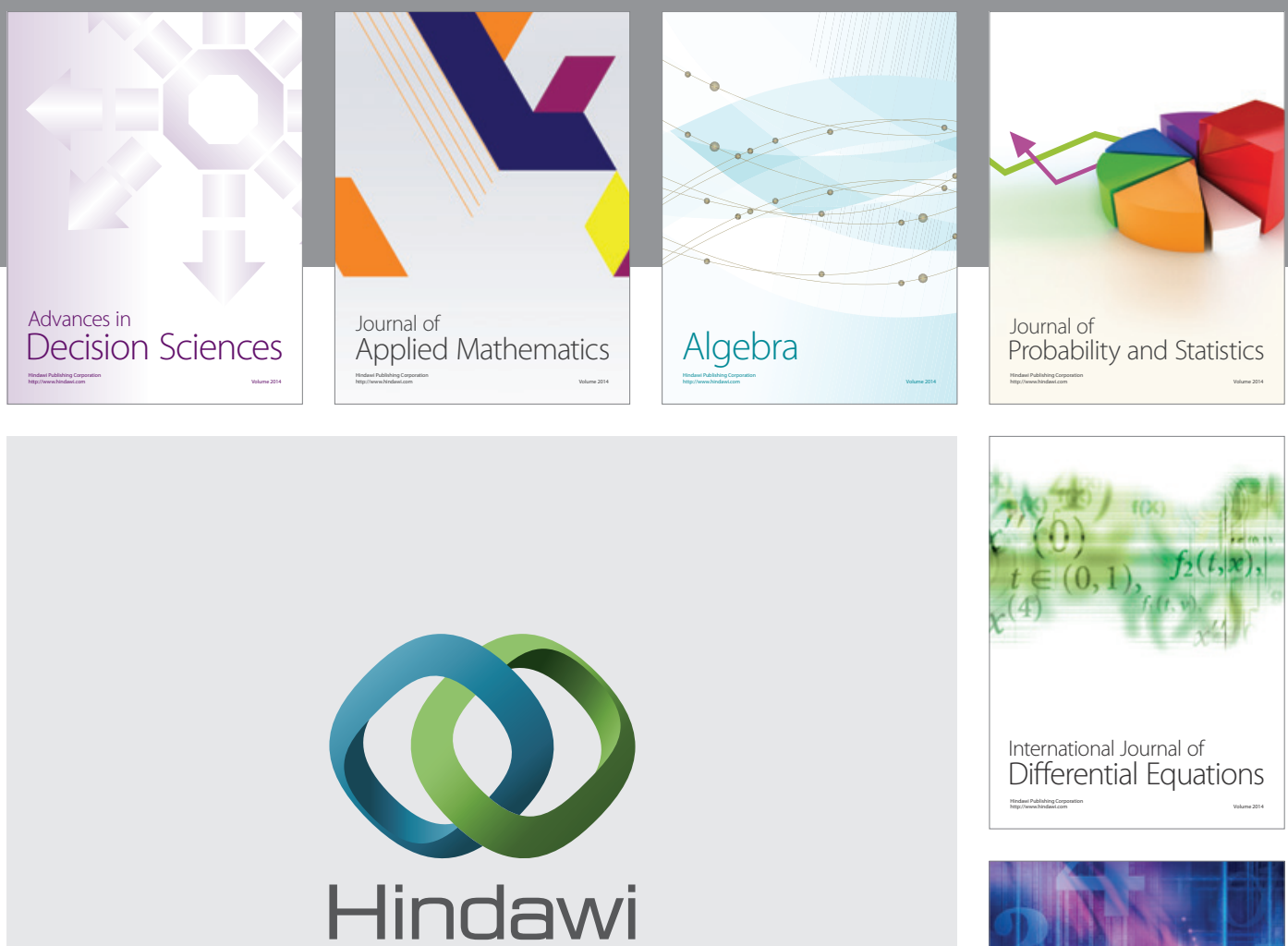

Submit your manuscripts at http://www.hindawi.com
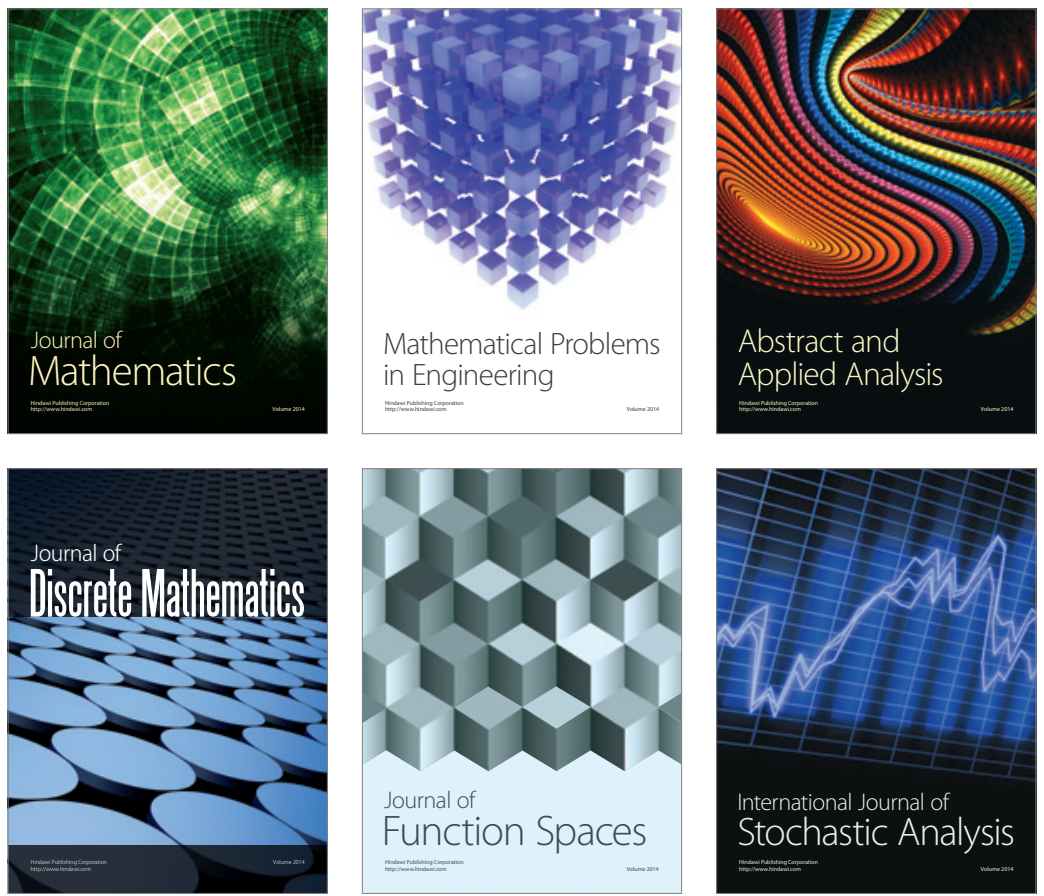

Journal of

Function Spaces

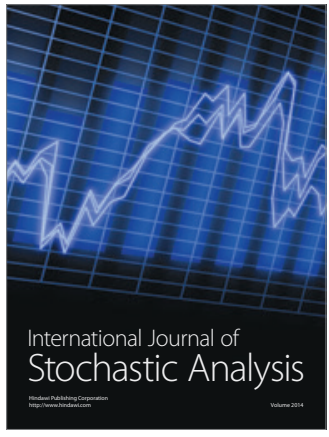

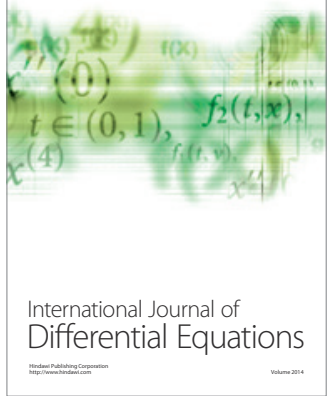
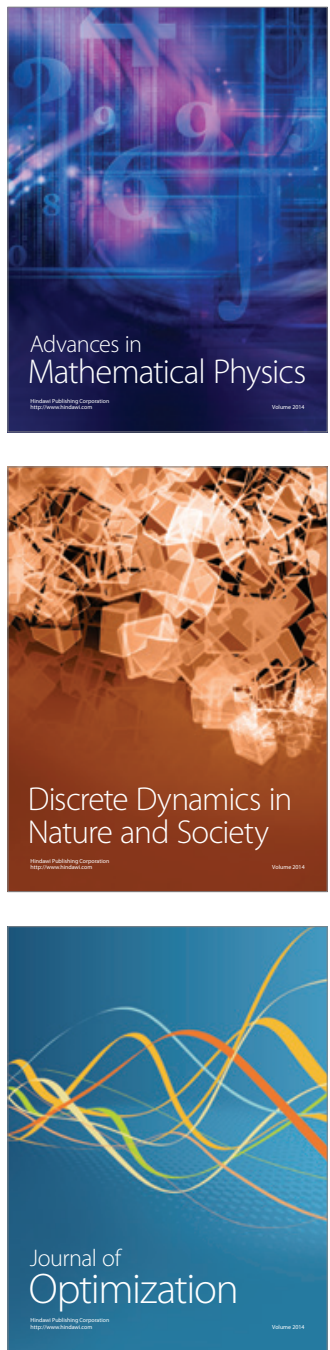